九州大学学術情報リポジトリ

Kyushu University Institutional Repository

\title{
ON THE STOCHASTIC DYNAMIC PROGRAMMING IN A STOCHASTIC SYSTEM AND THE MEASUREMENT OF A UTILITY FUNCTION OF AMOUNT OF MONEY IN THE SYSTEM
}

Kugimiya, Yasuo

Department of Economics, Oita University

https://doi.org/10.5109/13035

出版情報: 統計数理研究. 13 (3/4), pp. 15-55, 1969-03. Research Association of Statistical Sciences

バージョン :

権利関係 : 


\title{
ON THE STOCHASTIC DYNAMIC PROGRAMMING IN A STOCHASTIC SYSTEM AND THE MEASUREMENT OF A UTILITY FUNCTION OF AMOUNT OF MONEY IN THE SYSTEM
}

\author{
By \\ Yasuo KUGIMIYA*
}

(Received, Nov. 30, 1968)

\begin{abstract}
An analytic decision rule is developed for a problem of multi-stage stochastic strategy-making in a stochastic system. The problem of multi-stage stochastic strategy-making is characterized as making of an optimal sequence of strategic functions which is so called "stochastic dynamic programming."

On the basis of the stochastic dynamic programming, a theoretical survey will be conducted from a new angle of the measurement of a reasonable utility function of amount of money in the stochastic system. Furthermore, one of the reasons why the strategy maximizing expectation of amount of money produces contradiction will be illustrated. And it will be also illustrated that "Bernoulli's utility function" is not always applicable but it is applicable only through some modifications in the system.
\end{abstract}

The author wishes to express his hearty thanks to Professor T. Kitagawa for his continuous encouragement and guidance throughout this work.

\section{§1. Introduction}

Let us call employment of a strategy on a stochastic scheme "stochastic strategy-making." In multi-stage stochastic strategy-making, a decision is made at each stage on the basis of the information available at that stage. That information typically consists of realized values pertaining to the previous and current stages and expectations pertaining to the ensuing stages. Optimal strategy to such problem consists of (i) an optimal first stage decision, and (ii) an optimal decision rule for the additional stages. A decision rule may be denoted as a sequence of functions of certain variables on those stages. And a first stage decision also may be denoted by functions of the certain variables in combination with the realized values of the variables at that stage.

In this paper, a sequence of functions of amount of money which denotes a decision rule and each function of the sequence are called "strategic functions." And making of an optimal sequence of strategic functions (i.e. an optimal strategic function) from an initial time through an assigned last time in a stochastic system, on the basis of a criterion, is called "stochastic dynamic programming."

\footnotetext{
* Department of Economics, Oita University, Oita
} 
The problem of dynamic programming and the problem of stochastic dynamic programming have been studied extensively in recent years $[2,3,5,7,10,14,17$, etc. $]$. It may be generally agreed that the stochastic dynamic programming is conducted by (i) building a stochastic dynamic system, (ii) defining a feasible strategic function and determining the set of all feasible strategic functions in the system, and (iii) defining a reasonable criterion which measures degrees of effectiveness of any feasible strategic function in the system.

The problem of stochastic dynamic programming which we shall develop in this paper is characterized from the following points of view.

(1) In the stochastic dynamic system, a decision maker employs a sequence of functions of amount of money as his strategy. He chooses his actual strategy at each time as the value of strategic function on the amount of his money at that time, and obtains (positive or negative) money at the next time.

(2) In the system, the decision maker adds the obtained (positive or negative) money to the amount of his money. If the amount of his money reaches a lower bound at a time by means of the sequence of strategic functions, he cannot set his strategies at the ensuing times, and he is bankrupted.

(3) If (i) a lower bound of amount of money is assigned, and (ii) a decision maker has an amount of money at the initial time and he chooses a sequence of strategic functions, the amount of his money at each ensuing time can be forecast by the Markov process.

(4) A definition of feasible strategic function is given in connection with the bankruptcy.

(5) A reasonable criterion which measures degrees of effectiveness of a sequence of feasible strategic functions is made objectively from an economic point of view, by pursuing the Markov process produced by the sequence of strategic functions and the lower bound of amount of money.

Especially, in making a reasonable criterion, the following situations are noticed.

In many economic situations, a realistic formulation of the problem of stochastic dynamic programming frequently involves a large, possibly infinite, number of stages. And it may be safe to remember that the real last time of programming may be infinite future. However, in many cases, the need arises to truncate the many-stages or infinite stages after a " manageable" number of stages. The original problem is then replaced by a "proximate" problem involving finite stages. And no generally applicable criterion to such a "proximate" problem has yet been developed to the best of our knowledge.

Charnes, Drèze and Miller ${ }^{1)}$ state the following on the general theoretical manners in order to replace the original problem of a multi-stage stochastic strategy-making by a proximate problem.

The terminal conditions of the proximate problem somehow reflect the relevance of the future (beyond the truncation point) for immediate decisions. These conditions may, for instance, prescribe the terminal levels of certain variables of the system, or

1) See $[5]$ p. 308 . 
more generally assign "values" or "utilities" to such levels. Terminal conditions can be specified in various ways, namely,

(1st approach) by invoking constraints or value judg ments external to the immediate context of the problem;

(2nd approach) by establishing "convergence properties" and truncating the problem at a sufficiently remote point in time, so that solutions to the proximate problem will be "arbitrarily close to optimal";

(3rd approach) by deducing from an analysis of the original problem the properties that terminal conditions for the proximate problem must have, if an optimal solution to the proximate problem is to be consistent, under appropriate continuation, with an optimal solution to the original problem.

Now, there are many studies $[1,6,8,11,14,16$ etc. $]$ which define criterions for the stochastic strategy-making by means of a mathematical expectation of "utility of amount of money" (which is a value judgment) made from external of the immediate context of the problem. And, in recent years, for the purpose of stochastic strategy making, the problem of measurement of an actual utility function of amount of money has been studied extensively $[1,6,8,11,13,16$ etc. $]$.

However, even if a decision maker intends to employ a utility function of amount of money from external to the immediate context of the problem in making an optimal stochastic strategy, he must not employ the utility function only on the basis of his subjectivity. For instance, if a linear utility function is employed only on the basis of certain subjectivity, the strategy which maximizes mathematical expectation of utility of amount of money is equal to the strategy which maximizes mathematical expectation of amount of money. And it is agreed that the strategy which maximizes expectation of amount of money cannot always be optimal for the purpose of obtaining the maximal amount of money, such as the strategies in "Petersbrug's problem" and "problem of portfolio selection." Therefore, if a decision maker employs a linear utility function only on the basis of his subjectivity, in many cases, the utility function is not applicable.

In a multi-stage stochastic strategy making on the basis of a stochastic dynamic system, one must choose his utility function so that it may not contradict any objective reasonable conditions in the system. And some objective reasonable conditions for a utility function in a stochastic dynamic system are made from the points of view of the 3rd approach above. This paper intends to provide a modest contribution to a utility function which does not contradict some conditions which seem reasonable from the points of view of the 3rd approach above. Furthermore, it will be shown that the utility function has an important meaning also from the point of view of the 2nd approach.

We will begin by defining some fundamental notations (in Section 2). Then we will build a simple stochastic dynamic system which is a base of our arguments (in Section 3). And we define a feasible strategic function and develop a fundamental manner of the stochastic dynamic programming in the system (in Section 4).

Now, generally, the fundamental manner of the stochastic dynamic programming meets with serious difficulties. It is necessary to introduce some manners in order 
to evade the difficulties. The manners to evade the difficulties will be illustrated (in Sections 5 and 6).

Next, it is necessary to define a criterion which measures degree of effectiveness of each feasible strategic function, for the stochastic dynamic programming in the system defined in Section 3. It may seem reasonable that the criterion is defined on the basis of a utility function, from the point of view of the 1st approach above. However, we propose some assumptions concerning the utility function, which seems reasonable from the point of view of the $3 \mathrm{rd}$ approach in actual economic situations. And we will produce an analytic utility function which satisfies the proposed assumptions (in Section 7).

Furthermore, we see that the analytic utility function produced in Section 7 is really reasonable from the point of view of the 2nd approach, in the multi-stage stochastic strategy-making in the system defined in Section 3 (in Section 8). The theory in Section 8 will serve to point out many misuses of the "law of large numbers." And we see that the linear utility function and the Bernoulli's utility function [4] are not applicable in the system defined in Section 3, from some theoretical points of view in the stochastic dynamic programming (in Section 9).

\section{§2. Notation}

(i) $e_{t}(t=0,1,2, \cdots)$ denotes a variable noticed at a time $t$.

(ii) $B_{t}$ and $C_{t}(t=0,1,2, \cdots)$ denote certain stochastic variables realize themselves as $b_{t}$ and $c_{t}$, respectively. Probability density functions of the stochastic variables $B_{t}$ and $C_{t}$ are denoted by $\eta_{t}$ and $\phi_{t}$, respectively. And, the mathematical expectation of the stochastic variable $G\left(B_{t}\right)$ with density function $\eta_{t}$ is denoted by $E\left(G\left(B_{t}\right)\right.$ ), and

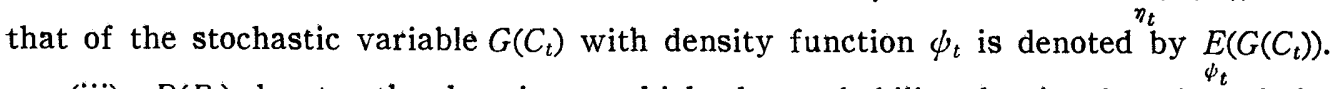

(iii) $D\left(B_{t}\right)$ denotes the domain on which the probability density function of the stochastic variable $B_{t}$ is positive.

(iv) If there are no confusions by omitting the suffix which assigns a time $t$, the suffix may be neglected, so that $C_{t}$ may be denoted by simple $C$.

(v) $D^{c}$ denotes the complement domain of a domain $D$.

(vi) Vector $\left(c_{n}, c_{n+1}, \cdots, c_{N}\right)$ is denoted by ${ }_{n}^{N} C$ or simple $C$. If we have $\beta<c_{t}<\gamma$ $(t=n, n+1, \cdots, N)$ with respect to two constants $\beta$ and $\gamma$, for short, all inequalities are denoted by $\beta<{ }_{n}^{N} C<\gamma$.

(vii) A sequence of functions $\left\{f_{n}\left(e_{n}\right), f_{n+1}\left(e_{n+1}\right), \cdots, f_{N}\left(e_{N}\right)\right\}$ are denoted by $F(n, N)$, and so on. If each component function $f_{t}\left(e_{t}\right)(t=n, n+1, \cdots, N)$ of $F(n, N)$ satisfies the relation $T\left(e_{t}\right) \leqq f_{t}\left(e_{t}\right)$ (or $T\left(e_{t}\right)=f_{t}\left(e_{t}\right)$ ) on a domain $D$ with respect to a function $T(e)$, for short, all relations are denoted by $T(e) \lesssim F(n, N)$ (or $T(e) \sim F(n, N)$ ) on the domain $D$, and so on.

(viii) $F(n, N ; \hat{F}(n, \infty))$ denotes the initial sub-sequence of $\hat{F}(n, \infty)$ composed of $N-n+1$ component functions. And $F(n, \infty ; \hat{F}(n, N))$ is an infinite sequence of functions in which the initial sub-sequence through the $N-n+1$-th function $f_{N}\left(e_{N}\right)$ coincides with $\hat{F}(n, N)$. 


\section{§ 3. System Building}

Let us build a system which is a foundation for our following argument. In the system, $b_{t}$ denotes a capital money, and $x_{t}$ and $y_{t}$ denote activity levels. A system is defined by the following conditions.

Condition 3.1. (i) A sequence of independent stochastic real variables $\left\{C_{0}, C_{1}, C_{2}\right.$, $\ldots\}$ is given. Each stochastic variable $C_{t}$ realizes itself as $c_{t}$ at the time $t+1(t=0,1,2$, $\cdots\}$.

(ii) All stochastic variables $C_{t}$ 's $(t=0,1,2, \cdots)$ have a common bounded probability density function $\phi_{t}$. And we have $0<\underset{\psi_{t}}{E}\left\{C_{t}\right\}=m<1$.

(iii) There are constants $\beta(0<\beta<1)$ and $\gamma(0<\gamma<1)$ which are independent of $t$, and for which (i) we have

$$
P\left\{\bigcap_{0 \leqq t}\left(-\beta<C_{t}<\gamma\right)\right\}=1 \quad(t=0,1,2, \cdots),
$$

and (ii) we have $\phi_{t}\left(e_{t}\right)>0$ on the domain $(-\beta, \gamma)$; that is, we have

$$
P\left\{-\beta+\varepsilon>C_{t}\right\} \geqq \delta>0 \quad(t=0,1,2, \cdots)
$$

with respect to any assigned positive number $\varepsilon$, in combination with a suitable chosen positive number $\delta$.

Condition 3.2. (i) Constants $K, M$ and $r$ and the initial amount of capital money $b_{0}$ are known by a decision maker at the initial time 0 , where $0<r<m<1$ and $M<\min \left(-K / r, b_{0}\right) .^{1)}$

(ii) The decision maker chooses a sequence of single valued non-negative functions $\left\{f_{0}\left(e_{0}\right), f_{1}\left(e_{1}\right), \cdots, f_{N}\left(e_{N}\right)\right\}$ (for short $F(0, N)$, which is defined on the direct product space $\prod_{t=0}^{N}\left\{e_{t} ; e_{t}>M\right\}$. Each function $f_{t}\left(e_{t}\right)$ is a continuous function on the domain $\left\{e_{t} ; e_{t}>M\right\}$ except finite discontinuous points.

$f_{t}\left(e_{t}\right)$ and $F(n, N)$ are called "strategic function."

(iii) The decision maker sets his strategic value as $x_{0}=f_{0}\left(b_{0}\right)$ and $y_{0}=b_{0}-x_{0}$ at the initial time 0 , and he earns an amount of money (which may be negative)

$$
g_{0}=c_{0} \cdot x_{0}+r \cdot y_{0}+K
$$

at the time 1 .

Condition 3.3. (i) At the time $t(t=1,2,3, \cdots)$, the decision maker knows the amount of capital money at that time, $b_{t}$.

(ii) If $b_{t}$ is greater than $M$, the decision maker sets his strategic values as $x_{t}$ $=f_{t}\left(b_{t}\right)$ and $y_{t}=b_{t}-x_{t}$ at the time $t$, and he earns an amount of money (which may be negative)

$$
g_{t}=c_{t} \cdot x_{t}+r \cdot y_{t}+K \quad(t=1,2,3, \cdots)
$$

at the time $t+1$. However, if $b_{t}$ is smaller than or equal to $M$, he cannot set his strategic value. That is, the decision maker suffers an exact loss $b_{t}-b_{0}\left(<M-b_{0}\right)$

1) This value $-K / r$ will play an important role in the following arguments. A practical meaning of this value will be illustrated in Section 5 . 
which cannot recover forever, and he is bankrupted.

CONDITION 3.4. If the amount of money $g_{t}$ is earned, the amount of money is added to $b_{t}$ at the time $t+1$, i.e. we have

$$
b_{t \div 1}=b_{t}+g_{t} \quad(t=0,1,2, \cdots) .
$$

In what follows, let us call the system defined by Conditions 3.1 through 3.4 "first type of stochastic dynamic management system," and denote the system by "S. D. M. S.-I."

In S. D. M.S.-I, we have the following fundamental stochastic relation

$$
\begin{gathered}
B_{t+1}=b_{t}+C_{t} \cdot f_{t}\left(b_{t}\right)+r \cdot\left(b_{t}-f_{t}\left(b_{t}\right)\right)+K=(1+r) \cdot b_{t}+\left(C_{t}-r\right) \cdot f_{t}\left(b_{t}\right)+K \\
\left(b_{t}>M ; t=0,1,2, \cdots\right) .
\end{gathered}
$$

Now, if (i) a decision maker has an amount of capital money $b_{n}$ and he chooses a strategic function $F(n, N)$ at a time $n$, and (ii) a sequence of realizations $\left\{c_{n}, c_{n+1}\right.$, $\left.c_{n+2}, \cdots, c_{N}\right\}$ realizes from a sequence of stochastic variables $\left\{C_{n}, C_{n+1}, \cdots, C_{N}\right\}$, then the amount of capital money at a time $s(n+1 \leqq s \leqq N+1), b_{s}$, is uniquely determined through successive application of the stochastic relation (3.6). Therefore, in what follows, let $B_{s}\left(b_{n}, F(n, N)\right)(n+1 \leqq s \leqq N+1)$ denote such a stochastic variable as may produce $b_{s}$ as its realization being subject to stochastic variables $C_{t}$ 's $(t=n, n+1$, $\cdots, s-1)$, on the basis of the capital money $b_{n}$ and the strategic function $F(n, N)$.

\section{§4. Fundamental Stochastic Dynamic Programming in S. D. M. S.-I.}

In order to carry out the stochastic dynamic programming in S.D.M.S.-I, first of all, let us define a feasible strategic function in S. D. M. S.-I.

DeFInition 4.1. Such strategic function $F(n, \infty)$ as the relations

$$
P\left\{\bigcup_{n+1 \leqq t}\left(B_{t} \leqq M\right) \mid b_{n}, F(n, \infty)\right\}=0, \quad F(n, \infty) \succsim 0
$$

hold true is called " $b_{n}$-feasible." 1)

A $b_{n}$-feasible strategic function is denoted by $F\left(n, \infty ; b_{n}\right) \equiv\left\{f_{n}\left(e_{n} ; b_{n}\right), f_{n+1}\left(e_{n+1}\right.\right.$; $\left.\left.b_{n}\right), f_{n+2}\left(e_{n \div 2} ; b_{n}\right), \cdots\right\}$ and the set of all $b_{n}$-feasible strategic functions is denoted by $\mathfrak{\mho}\left(n, \infty ; b_{n}\right)$.

DEFINITION 4.2. An element of the set $\bigcap_{n=D} \mathfrak{F}\left(n, \infty ; b_{n}\right)$ is called "D-feasible."

A $D$-feasible strategic function is denoted by $F(n, \infty ; D) \equiv\left\{f_{n}\left(e_{n} ; D\right), f_{n+1}\left(e_{n+1} ; D\right)\right.$, $\cdots\}$, and the set $\bigcap_{b_{n} \in D} \mathfrak{F}\left(n, \infty ; b_{n}\right)$ is denoted by $\mathfrak{F}(n, \infty ; D)$.

Definition 4.3. Let $\phi$ denote the empty set. If a strategic function $F(n, N)$ satisfies the following two conditions

1) In actual economic situations, such an attitude with respect to a feasible strategic function seems to be too strict, since any entrepreneur cannot carry out his own management activity without a certain amount of probability of going bankrupt. However, such an assumed attitude in making strategic function does not restrict the generality of argument in order to apply the attitude to an actual stochastic strately-making. If a decision maker admits the risk of going bankrupt to a certain amount of probability, our following argument can be modified according to the amount of risk he admits. 


$$
P\left\{\bigcup_{n \rightarrow 1 \leqq t \leqq N+1}\left(B_{t} \leqq M\right) \mid b_{n}, F(n, N)\right\}=0, \quad F(n, N) \gtrsim 0
$$

and

$$
\mathfrak{F}\left(N+1, \infty ; D\left(B_{N+1}\left(b_{n}, F(n, N)\right)\right)\right) \neq \phi,
$$

then the strategic function $F(n, N)$ is called " $b_{n}$-feasible."

A $b_{n}$-feasible strategic function is denoted by $F\left(n, N ; b_{n}\right) \equiv\left\{f_{n}\left(e_{n} ; b_{n}\right), f_{n+1}\left(e_{n+1} ; b_{n}\right)\right.$, $\left.\cdots, f_{N}\left(e_{N} ; b_{n}\right)\right\}$ and the set of all $b_{n}$-feasible strategic functions is denoted by $\mathfrak{F}\left(n, N ; b_{n}\right)$.

DeFinition 4.4. An element of the set $\bigcap_{b_{n}=D} \mathfrak{F}\left(n, N ; b_{n}\right)$ is called "D-feasible."

A $D$-feasible strategic function is denoted by $F(n, N ; D) \equiv\left\{f_{n}\left(e_{n} ; D\right), f_{n \div 1}\left(e_{n+1} ; D\right)\right.$, $\left.\cdots, f_{N}\left(e_{N} ; D\right)\right\}$, and the set $\bigcap_{b_{n}=D} \mathfrak{F}\left(n, N ; b_{n}\right)$ is denoted by $\mathfrak{F}(n, N ; D)$.

DEFINITION 4.5. If a steategic function is $D\left(B_{n}\right)$-feasible with respect to a stochastic variable $B_{n}$, the strategic function is also called " $B_{n}$-feasible."

A $B_{n}$-feasible strategic function is denoted by $F\left(n, \infty ; B_{n}\right) \equiv\left\{f_{n}\left(e_{n} ; B_{n}\right), f_{n+1}\left(e_{n+1}\right.\right.$; $\left.\left.B_{n}\right), f_{n+2}\left(e_{n+2} ; B_{n}\right), \cdots\right\}$ or $F\left(n, N ; B_{n}\right) \equiv\left\{f_{n}\left(e_{n} ; B_{n}\right), f_{n+1}\left(e_{n+1} ; B_{n}\right), \cdots, f_{N}\left(e_{N} ; B_{n}\right)\right\}$. And the set of all $B_{n}$-feasible strategic functions is denoted by $\mathfrak{F}\left(n, \infty, B_{n}\right)$ or $\mathfrak{F}\left(n, N ; B_{n}\right)$.

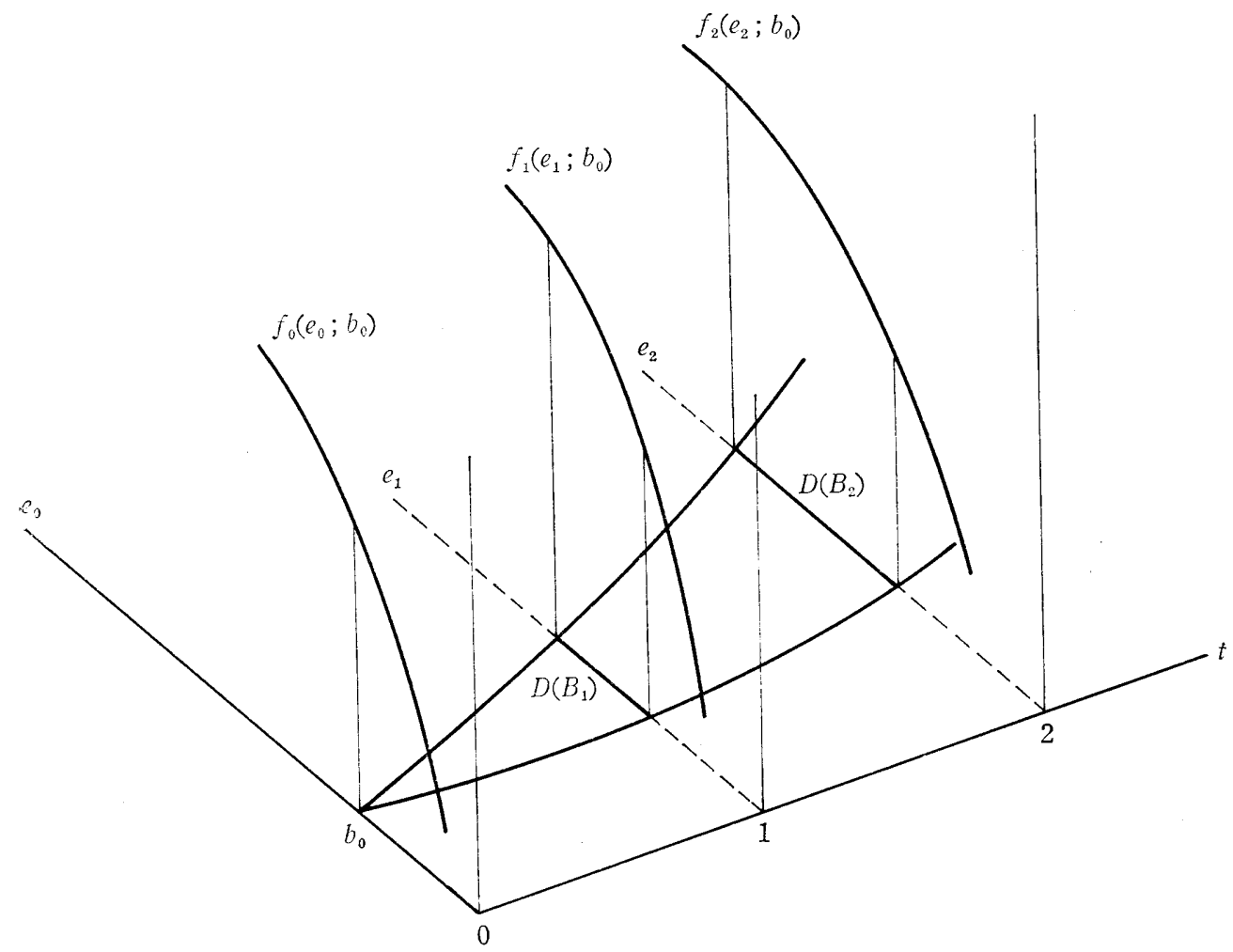

Fig. 4.1.

Next, let us define a criterion which measures the degree of effectiveness of any $b_{n}$-feasible strategic function in S.D. M.S.-I. According to the point of view of the first approach in Section 1, let us employ the following: 
DEFINITION 4.6. At a time $n$, if (i) a decision maker has an amount of capital money $b_{n}$, (ii) he assigns the last time of programming $N$, and (iii) he chooses a utility function of amount of money $\xi(\mathrm{e})$, then the functional

$$
U\left(F\left(n, N ; b_{n}\right) ; b_{n}, \xi\right) \equiv \frac{1}{N-n+1} \cdot\left[\underset{n_{N+1}}{E}\left\{\xi\left(B_{N+1}\right) \mid b_{n}, F\left(n, N ; b_{n}\right)\right\}-\xi\left(b_{n}\right)\right]
$$

defined on $\mathfrak{F}\left(n, N ; b_{n}\right)$ is called a “ $\left(b_{n}, \xi\right)$-criterion functional," and a functional $\lim _{N \rightarrow \infty} U\left(F\left(n, N ; F\left(n, \infty ; b_{n}\right)\right) ; b_{n}, \xi\right)$ defined on $\mathfrak{F}\left(n, \infty ; b_{n}\right)$ is also called a " $\left(b_{n}, \xi\right)$-criterion functional."

DeFinition 4.7. In S.D.M.S.-I, if (i) the $\left(b_{n}, \xi\right)$-criterion functional $U\left(F\left(n, N ; b_{n}\right)\right.$; $\left.b_{n}, \xi\right)$ (or $U\left(F\left(n, \infty ; b_{n}\right) ; b_{n}, \xi\right)$ ) defined on $\mathfrak{F}\left(n, N ; b_{n}\right)\left(\right.$ or $\mathfrak{F}\left(n, \infty ; b_{n}\right)$ ) is finite or negative infinite, and (ii) a strategic function $\hat{F}\left(n, N ; b_{n}\right)\left(\right.$ or $\left.\hat{F}\left(n, \infty ; b_{n}\right)\right)$ maximizes the $\left(b_{n}, \xi\right)$-criterion functional $U\left(F\left(n, N ; b_{n}\right) ; b_{n}, \xi\right)\left(\right.$ or $\left.U\left(F\left(n, \infty ; b_{n}\right) ; b_{n}, \xi\right)\right)$ in $\mathfrak{F}\left(n, N ; b_{n}\right)$ (or $\left.\mathfrak{F}\left(n, \infty ; b_{n}\right)\right)$, then the strategic function $\hat{F}\left(n, N ; b_{n}\right)\left(\right.$ or $\left.\hat{F}\left(n, \infty ; b_{n}\right)\right)$ is called " $\left(b_{n}, \xi\right)$ optimal." 1$)$

In what follows, a $\left(b_{n}, \xi\right)$-optimal strategic function is denoted by $F^{*}\left(n, N ; b_{n}, \xi\right)$ (or $\left.F^{*}\left(n, \infty ; b_{n}, \xi\right)\right)$, and the set of all $\left(b_{n}, \xi\right)$-optimal strategic functions is denoted by $\mathfrak{F}^{*}\left(n, N ; b_{n}, \xi\right)$ (or $\mathfrak{F}^{*}\left(n, \infty ; b_{n}, \xi\right)$ ).

As we saw in Section 1, the original problem of the stochastic dynamic programming in S. D. M. S.-I may be making of a $\left(b_{0}, \xi\right)$-optimal strategic function $F *(0, \infty$; $\left.b_{0}, \xi\right)$. However, the need arises to truncate the infinite stages after a "manageable" number of stages, and to make a $\left(b_{0}, \xi\right)$-optimal strategic function $F^{*}\left(0, N ; b_{0}, \xi\right)$.

Now, if a $B_{n}$-feasible strategic function $F\left(n, N ; B_{n}\right)$ and a utility function $\xi(e)$. are assinged, $U\left(F\left(n, N ; B_{n}\right) ; e_{n}, \xi\right)$ may be a function of $e_{n}$ on the domain $D\left(B_{n}\right)$. We can define a stochastic variable $U\left(F\left(n, N ; B_{n}\right) ; B_{n}, \xi\right)$ which will realizes itself as $U\left(F\left(n, N ; B_{n}\right) ; b_{n}, \xi\right)$. Hence, we can calculate the mathematical expectation $E\left\{U\left(F\left(n, N ; B_{n}\right) ; B_{n}, \xi\right)\right\}$ if a stochastic variable $B_{n}$ and a $B_{n}$-feasible strategic function $F\left(n, N ; B_{n}\right)$ are assigned. That is, if a stochastic variable $B_{n}$ is assigned and a utility function $\xi(e)$ is employed, then $\operatorname{En}_{n_{n}}\left\{U\left(F\left(n, N ; B_{n}\right) ; B_{n}, \xi\right)\right\}$ is also a functional defined on the set $\mathfrak{F}\left(n, N ; B_{n}\right)$. We have the following

Definition 4.8. The functional ${ }_{\eta_{n}}\left\{U\left(F\left(n, N ; B_{n}\right) ; B_{n}, \xi\right)\right\}$, defined on the basis of (4.4) being connected with a stochastic variable $B_{n}$, is called a " $\left(B_{n}\right.$, $\left.\xi\right)$-criterion functional."

DEFINITION 4.9. In S. D.M.S.- $I$, if (i) the $\left(B_{n}, \xi\right)$-criterion functional $\underset{n_{n}}{E}\{U(F(n, N$; $\left.\left.B_{n}\right) ; B_{n}, \xi\right)$ is finite or negative infinite on $\mathfrak{F}\left(n, N ; B_{n}\right)$, and (ii) a strategic function $\hat{F}\left(n, N ; B_{n}\right)$ maximizes the $\left(B_{n}, \xi\right)$-criterion functional $\underset{n_{n}}{E}\left\{U\left(F\left(n, N ; B_{n}\right) ; B_{n}, \xi\right)\right\}$ in $\widetilde{F}\left(n, N ; B_{n}\right)$, then the strategic function $\hat{F}\left(n, N ; B_{n}\right)$ is called " $\left(B_{n}, \xi\right)$-optimal."

1) In S.D.M.S.-I, if (i) each stochastic variable $C_{t}(t=0,1,2, \cdots)$ has not any positive variance contradicting Condition 3.1, and (ii) there are no restrictions with respect to the upper bound of $f_{t}\left(b_{t}\right)$, then any values of optimal strategic function at each time may be equal to 0 in the case of $c_{t}-r<0$, and the values may be positive infinite in the case of $c_{t}-r>0$. However, if $C_{t}$ 's are stochastic variables which have positive variance, the stochastic dynamic programming is not always so simple. 
In what follows, a $\left(b_{n}, \xi\right)$-optimal strategic function is denoted by $F^{*}\left(n, N ; B_{n}, \xi\right)$, and the set of all $\left(B_{n}, \xi\right)$-optimal strategic function is denoted by $\mathfrak{F}^{*}\left(n, N ; B_{n}, \xi\right)$.

Applying the principle of optimality in dynamic programming which is generalized on S. D. M. S.-I, in connection with (4.4), we have

$$
\begin{aligned}
& \underset{\eta_{n}}{E}\left\{U\left(F *\left(n, N ; B_{n}, \xi\right) ; B_{n}, \xi\right)\right\}=\max _{F\left(n, N ; B_{n}\right)} \underset{\eta_{n}}{E}\left\{U\left(F\left(n, N ; B_{n}\right) ; B_{n}, \xi\right)\right\} \\
&=\frac{1}{N-n+1} \cdot \max _{f_{n}\left(e_{n} ; B_{n}\right)} \underset{\eta_{n}}{E}\left[U\left(f_{n}\left(e_{n} ; B_{n}\right) ; B_{n}, \xi\right)\right. \\
&+(N-n) \cdot \max _{F\left(n+1, N ; B_{n+1}\left(B_{n}, f_{n}\left(e_{n} ; B_{n}\right)\right)\right)} \underset{\eta_{n+1}}{E}\left\{U\left(F\left(n+1, N ; B_{n+1}\right) ; B_{n+1}, \xi\right)\right. \\
&= \frac{1}{N-n+1} \cdot \max _{f_{n}\left(e_{n} ; B_{n}\right)} E\left[U \left(f_{n}\left(f_{n}\left(e_{n} ; B_{n}\right) ; B_{n}, \xi\right)\right.\right. \\
&+(N-n) \cdot \underbrace{E}_{\eta_{n-1}}\left\{U\left(F^{*}\left(n+1, N ; B_{n+1}, \xi\right) ; B_{n+1}, \xi\right) \mid B_{n}, f_{n}\left(e_{n} ; B_{n}\right)\right\}] \\
& \quad\left(n=0,1,2, \cdots, N ; B_{0}=b_{0}\right) .
\end{aligned}
$$

\section{§. A Special Domain $\bar{D}$ and the Set of All $\bar{D}$-feasible strategic functions}

Now, if we intend to obtain a $\left(b_{0}, \xi\right)$-optimal strategic function $F^{*}\left(0, N ; b_{0}, \xi\right)$ in S. D. M. S.-I by means of the forward working algorithm on the basis of the relation (4.5), it is, at least, necessary (i) to purse the domain $D\left(B_{n}\left(b_{0}, F\left(0, N ; b_{0}\right)\right)\right.$ with respect to each $n(n=1,2, \cdots, N)$ and each $F\left(0, N ; b_{0}\right)$ in $\mathscr{F}\left(0, N ; b_{0}\right)$, and (ii) to verify that either a $\left(b_{n}, \xi\right)$-optimal strategic function $F^{*}\left(n, N ; b_{n}, \xi\right)$ can or cannot be chosen with respect to each $n(n=0,1,2, \cdots, N)$ and to any $b_{n}$ on the domain $D\left(B_{n}\left(b_{0}\right.\right.$, $\left.\left.F^{*}\left(0, N ; b_{0}, \xi\right)\right)\right)$. Therefore, if we intend to conduct the stochastic dynamic programming in S. D. M. S.-I directly by means of the stochastic relation (4.5), the derivation and computation of an optimal solution meets with considerable difficulties. One must thus simplify the problem of stochastic dynamic programming so that the forward working algorithm may be applied more easily.

For our purpose, first of all, let us introduce special domains $\widetilde{D}(n, N)$ 's by the following

DeFinition 5.1. Let $\phi$ be the empty set. Such domain $\bar{D}(n, N)($ or $\bar{D}(n ; \infty))$ as the following two conditions

$$
\mathfrak{F}(n, N ; \bar{D}(n, N)) \neq \phi \quad(\text { or } \mathfrak{F}(n, \infty ; \bar{D}(n, \infty)) \neq \phi)
$$

and

$$
\bigcup_{b_{n}=\bar{D}(n, N) c} \mathfrak{F}\left(n, N ; b_{n}\right)=\phi \quad\left(\text { or } \underset{b_{n} \in \bar{D}(n, \infty) c}{\bigcup} \mathfrak{F}\left(n, \infty ; b_{n}\right)=\phi\right)
$$

hold true is called a "maximal fuasible domain" pertaining to $n$ and $N$ (or $n$ and $\infty$ ).

Then, we have the following

THEOREM 5.1. If there is the domain $\bar{D}(n, N)$ pertaining to any assigned nonnegative integers $n$ and $N$ such that $n \leqq N$, these domains $\bar{D}(n, N)$ 's are independent of $n$ and $N$ in S. D. M.S.-I. 
Proof. If there is a $b_{n}$-feasible strategic function $\hat{F}\left(n, \infty ; b_{n}\right)$, the strategic function $F\left(n, N ; \hat{F}\left(n, \infty ; b_{n}\right)\right)$ is also a $b_{n}$-feasible strategic function, according to Definitions 4.1 and 4.3. Therefore, we have

$$
\bar{D}(n, \infty) \leqq \bar{D}(n, N) .
$$

Furthermore, if there is a $b_{n}$-feasible strategic function $\hat{F}\left(n, N ; b_{n}\right)$, we can also choose such a $b_{n}$-feasible strategic function $\hat{F}\left(n, \infty ; b_{n}\right)$ as

$$
\hat{F}\left(n, N ; b_{n}\right) \equiv F\left(n, N ; \hat{F}\left(n, \infty ; b_{n}\right)\right)
$$

holds true according to Definitions 4.1 and 4.3. Hence, we have

$$
\bar{D}(n, N) \subseteq \bar{D}(n, \infty) \text {. }
$$

According to (5.3) and (5.5), we have $\bar{D}(n, N)=\bar{D}(n, \infty)$. And, since the stochastic process $\left\{C_{0}, C_{1}, C_{2}, \cdots\right\}$ is a stationary stochastic process in S.D.M.S.-I, the domain $\bar{D}(n, \infty)$ is independent of $n$, and the theorem is verified.

Q.E.D.

Hence, we have the following

Definition 5.2. We call the domain $\bar{D}(0, \infty)$ the "maximal feasible domain," and denote the domain as simple $\bar{D}$.

Let us verify that (i) there is the maximal feasible domain $\bar{D}$, and that (ii) we can determine the concrete structure of the set of all $\bar{D}$-feasible strategic functions $\widetilde{\mho}(n, N$; $\bar{D})$, in S. D. M.S.-I. First, let us verify the following:

THEOREM 5.2. In S. D. M.S. $-I$, if $-K / r>b_{0}(>M)$, there are no $b_{0}$-feasible strategic function $F\left(0, \infty ; b_{0}\right)$, and also no $b_{0}$-feasible strategic function $F\left(0, N ; b_{0}\right)(N=0$, $1,2, \cdots)$.

PROOF. If a decision maker has capital money $b_{t}$ and he employs a strategic function $f_{t}\left(e_{t}\right)$ at a time $t$, the following relation

$$
\begin{aligned}
B_{t+1}\left(b_{t}, f_{t}\left(e_{t}\right)\right)+K / r & =(1+r) \cdot b_{t}+\left(C_{t}-r\right) \cdot f_{t}\left(b_{t}\right)+K+K / r \\
& =(1+r) \cdot\left(b_{t}+K / r\right)+\left(C_{t}-r\right) \cdot f_{t}\left(b_{t}\right) \quad(t=0,1,2, \cdots),
\end{aligned}
$$

is obtained, according to the fundamental stochastic relation (3.6).

Let us assume that $b_{0}<-K / r$ and a strategic function $\hat{F}(0, \infty) \equiv\left\{\hat{f}_{0}\left(e_{0}\right), \hat{f}_{1}\left(e_{1}\right)\right.$, $\left.\hat{f}_{2}\left(e_{2}\right), \cdots\right\}$ is a $b_{0}$-feasible strategic function. Then, we have the relation

$$
\begin{aligned}
& B_{n}\left(b_{n}, \hat{F}(0, \infty)+K / r\right. \\
&=(1+r)^{s} \cdot\left(b_{0}+K / r\right)+\sum_{t=0}^{s-1}(1+r)^{s-t-1} \cdot\left(C_{t}-r\right) \cdot \hat{f}_{t}\left(B_{t}\left(b_{0}, \hat{F}(0, \infty)\right)\right. \\
&\left(s=1,2,3, \cdots ; B_{0}=b_{0}\right),
\end{aligned}
$$

applying the relation (5.6) successively.

And, even if any constant $M$ is assigned, we can choose such an integer $n$ as the relation

$$
(1+r)^{n} \cdot\left(b_{0}+K / r\right) \leqq M+K / r
$$

holds true in the case of $b_{0}+K / r<0$, since we have $r>0$ from Condition 3.2-(i).

Furthermore, according to Condition 3.1-(iii), we can choose such a positive number $\mu$ as 


$$
P\left\{C_{t}-r<0\right\}=\mu>0 \quad(t=0,1,2, \cdots)
$$

holds true. And, of course, we have the relation

$$
P\left\{\hat{f}_{t}\left(B_{t}\right) \geqq 0 \mid b_{0}, \hat{F}(0, \infty)\right\}=1 \quad(t=0,1,2, \cdots)
$$

which, in combination of (5.9), yields us

$$
P\left\{\sum_{t=0}^{n-1}(1+r)^{n-t-1} \cdot\left(C_{t}-r\right) \cdot \hat{f}_{t}\left(B_{t}\right) \leqq 0 \mid b_{0}, \hat{F}(0, \infty)\right\} \geqq \mu^{n}>0
$$

with respect to the integer $n$ chosen in (5.8).

Connecting the relations (5.8) and (5.11) with the relation (5.7), we have

$$
\begin{aligned}
P\left\{B_{n}\right. & \left.\leqq M \mid b_{0}<-K / r, \hat{F}(0, \infty)\right\} \\
& =P\left\{B_{n}+K / r \leqq M+K / r \mid b_{0}<-K / r, \hat{F}(0, \infty)\right\} \\
& \geqq \mu^{n}>0 .
\end{aligned}
$$

This relation shows that the strategic function $\hat{F}(0, \infty)$ cannot be any $b_{0}$-feasible strategic function in the case of $b_{0}<-K / r$, and we have a contradiction. Q.E.D.

COROLlary 5.2.1. In S. D. M.S.-I, if there is a $\bar{D}$-feasible strategic function, any element of the domain $\bar{D}$ is not smaller than $-K / r$.

Corollary 5.2.2. In S.D.M.S. $-I$, if $P\left\{B_{t}<-K / r \mid b_{0}, \hat{F}(0, N)\right\}>0$ at a certain time $t(t=0,1,2, \cdots, N)$, the strategic function $\hat{F}(n, N)$ is not $b_{0}$-feasible.

PRoof. According to the assumption of this corollary, we can choose such two positive numbers $\varepsilon$ and $\delta$ as

$$
P\left\{B_{t} \leqq-K / r-\varepsilon \mid b_{0}, \hat{F}(0, N)\right\}>\delta>0 \quad(0 \leqq t \leqq N)
$$

holds true. And by means of the same manner as we have obtained (5.12), we can choose a positive number $\nu$ and a positive integer $n$ such that the relation

$$
P\left\{B_{t+n} \leqq M \mid b_{t} \leqq-K / r-\varepsilon, F(0, \infty ; \hat{F}(0, N))\right\}>\nu^{n}>0
$$

holds true.

Hence, we have

$$
P\left\{B_{t+n} \leqq M \mid b_{0}, F(0, \infty ; \hat{F}(0, N))\right\}>\delta \cdot \nu^{n}>0
$$

which shows the strategic function $\hat{F}(0, N)$ cannot be $b_{0}$-feasible, (see Fig. 5.1). Q. E. D.

COROLlaRY 5.2.3. We have $f_{t}\left(-K / r ; b_{n}\right)=0 \quad\left(b_{n} \geqq-K / r\right)$ with respect to each $t(t=n, n+1, \cdots, N)$.

Proof. According to (3.6), we have

$$
\begin{aligned}
B_{t+1}\left(b_{t}\right. & \left.=-K / r, f_{t}\left(e_{t} ; b_{n}\right)\right)+K / r \\
& =(1+r) \cdot(-K / r)+\left(C_{t}-r\right) \cdot f_{t}\left(-K / r ; b_{n}\right)+K+K / r \\
& =\left(C_{t}-r\right) \cdot f_{t}\left(-K / r ; b_{n}\right) \quad(t=n, n+1, \cdots, N) .
\end{aligned}
$$

On the other hand, there is such a positive number $\delta$ as $P\left\{C_{t}-r<-r\right\}>\delta>0$ holds true, from Condition 3.1-(iii). Since the relation $B_{t+1}\left(b_{t}=-K / r, f_{t}\left(e_{t} ; b_{n}\right)\right)+K / r$ $=\left(C_{t}-r\right) \cdot f_{t}\left(-K / r ; b_{n}\right) \geqq 0$ must be held with probability 1 from Corollary 5.2.2, $f_{t}(-K / r)$ must be equal to 0 according to (5.16).

Q.E.D. 


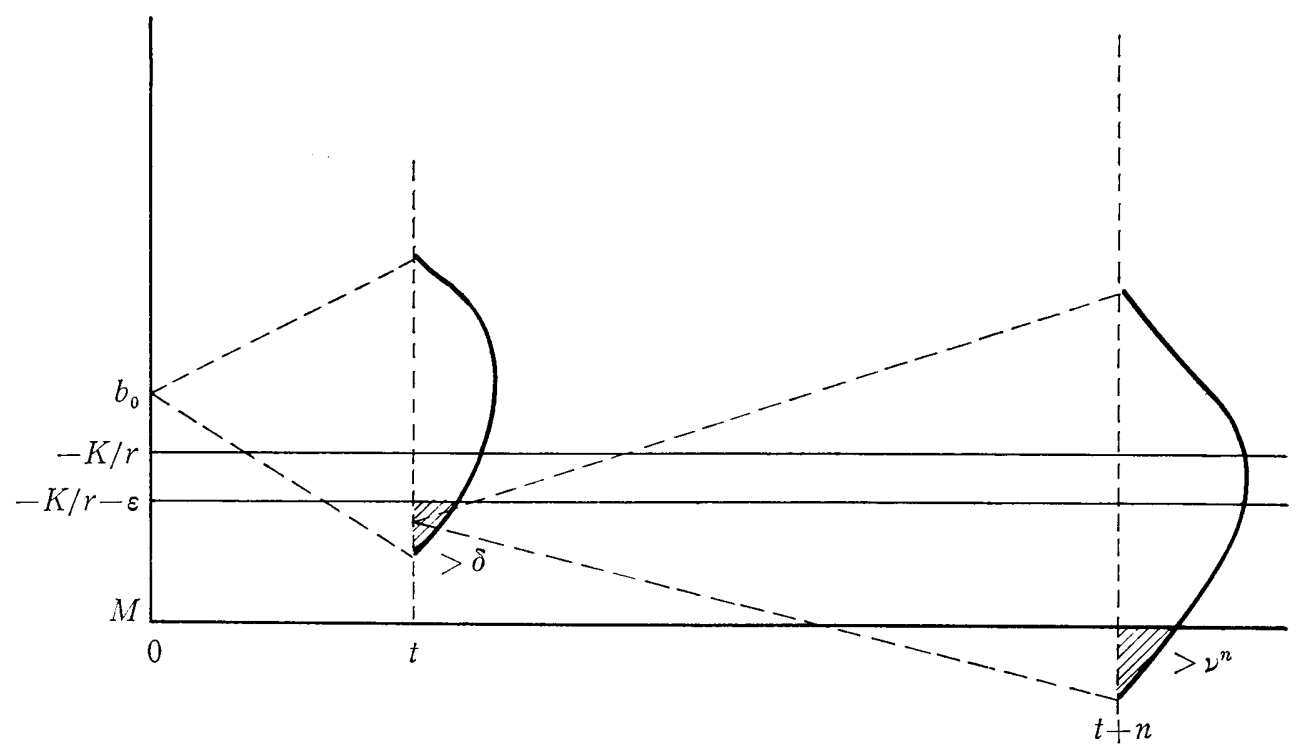

Fig. 5.1.

THEOREM 5.3. Let $\beta$ be the constant defined iu Condition 3.1-(iii). In S. D. M.S.-1, if a strategic function $F(0, \infty)$ satisfies the relation

$$
0 \lesssim F(0, \infty) \lesssim(1+r) \cdot(e+K / r) /(\beta+r)
$$

on the domain $[-K / r, \infty)$, then we have also

$$
P\left\{\bigcap_{1 \geqq n}\left(B_{n} \geqq-K / r\right) \mid b_{0} \geqq-K / r, F(0, \infty)\right\}=1 .
$$

Proof. In order to prove this theorem, as the first step, let us verify the following LEMmA 5.3.1. In S.D.M.S.-I, we have

$$
P\left\{B_{t+1} \geqq-K / r \mid b_{t} \geqq-K / r, 0 \leqq f_{t}\left(e_{t}\right) \leqq(1+r) \cdot\left(e_{t}+K / r\right) /(\beta+r)\right\}=1 .
$$

Proof of Lemma 5.3.1. We have $P\left\{-\beta<C_{t}<\gamma\right\}=1$ from (3.1). Therefore, if $f_{t}\left(e_{t}\right) \geqq 0$, we have

$$
P\left\{(1+r) \cdot\left(b_{t}+K / r\right)+\left(C_{t}-r\right) \cdot f_{t}\left(b_{t}\right) \geqq(1+r) \cdot\left(b_{t}+K / r\right)+(-\beta-r) \cdot f_{t}\left(b_{t}\right)\right\}=1 .
$$

Furthermore, if $b_{t}+K / r \geqq 0$ and $0 \leqq f_{t}\left(b_{t}\right) \leqq(1+r) \cdot\left(b_{t}+K / r\right) /(\beta+r)$, we have

$$
(1+r) \cdot\left(b_{t}+K / r\right)+(-\beta-r) \cdot f_{t}\left(b_{t}\right) \geqq 0,
$$

since $\beta+r>0$.

Applying the relations (5.20) and (5.21) to the relation (5.6), we can admit the relation (5.19).

LEMMA 5.3.1. Q. E. D.

If we apply the mathematical induction to (5.19), we can admit this theorem.

Q.E. D.

COROLLARY 5.3.1. The maximal feasible domain $\bar{D}$ (defined by Definition 5.2) is the domain $[-K / r, \infty)$.

PROOF. According to Corollary 5.2.1 and Theorem 5.3, we can admit this corollary.

Q.E.D. 
COROLlaRY 5.3.2. In S.D.M.S.- I, if a strategic function $F(n, N)$ satisfies the relation

$$
0 \lesssim F(n, N) \lesssim(1+r) \cdot(e+K / r) /(\beta+r)
$$

on the domain $\bar{D}$ (defined in Corollary 5.3.2), the steategic function is $\bar{D}$-feasible.

COROLLARY 5.3.3. If a strategic function $F(n, N)$ satisfies the relation (5.22), we have

$$
P\left\{\bigcap_{n \div 1 \leqq t \leqq N-1}\left(B_{t}>-K / r\right) \mid b_{n}>-K / r, F(n, N)\right\}=1 .
$$

The number $N$ may be substituted by $\infty$.

Theorem 5.3 (and Corollary 5.3.2) shows that the relation (5.17) (and (5.22)) is sufficient condition in order that the strategic function $F(0, \infty)$ (and $F(0, N)$ ) is $\bar{D}$. feasible. Furthermore, let us verify that this condition is also necessary condition.

Now, in the following many cases, we employ the following notation.

$$
\begin{aligned}
\frac{f_{t}\left(e_{t}\right)}{e_{t}+K / r}=h_{t}\left(e_{t}\right), \quad H(n, N) \equiv & \left\{h_{n}\left(e_{n}\right), h_{n+1}\left(e_{n+1}\right), \cdots, n_{N}\left(e_{N}\right)\right\} \\
& \left(e_{t} \in \bar{D} ; t=n, n+1, \cdots, N\right) .
\end{aligned}
$$

Then, each function $h_{t}\left(e_{t}\right)$ is uniquely determined on the domain $(-K / r, \infty)$ according to the function $f_{t}\left(e_{t}\right)$. And it is a single valued continuous function on the domain $(-K / r, \infty)$ except finite points.

$h_{t}(-K / r)$ may be any finite value since we have $f_{t}(-K / r)=0$ from Corollary 5.2.3. However, for the sake of simplicity, let us assume as follows:

$$
\lim _{t_{t} \rightarrow-K / r} h_{t}\left(e_{t}\right)=h_{t}(-K / r) \quad(t=0,1,2, \cdots) .
$$

Then, $h_{t}\left(e_{t}\right)$ is uniquely determined from the function $f_{t}\left(e_{t}\right)$ on the domain $\bar{D}$, and it is a single valued continuous function on that domain except finite points. In what follows, let us denote as $f_{t}\left(e_{t} ; \bar{D}\right) /\left(e_{t}+K / r\right)=h_{t}\left(e_{t} ; \bar{D}\right)$, and denote as $H(n, N ; \bar{D})$ $\equiv\left\{h_{n}\left(e_{n} ; \bar{D}\right), h_{n+1}\left(e_{n+1} ; \bar{D}\right), \cdots, h_{N}\left(e_{N} ; \bar{D}\right)\right\}$. Then, we have the relation

$$
0 \lesssim H(n, N ; \bar{D}) \lesssim(1+r) /(\beta+r)
$$

from (5.22) and (5.24).

Furthermore, applying the relation (5.24) to (3.6), we have a fundamental stochastic relation

$$
B_{t+1}+K / r=\left(b_{t}+K / r\right) \cdot\left(1+r+\left(C_{t}-r\right) \cdot h\left(b_{t}\right)\right) \quad\left(b_{t} \geqq-K / r, t=0,1,2, \cdots\right) .
$$

And if $b_{n} \in \bar{D}$, we have the following stochastic relation

$$
\begin{gathered}
B_{t}\left(b_{n}, F(n, N ; \bar{D})\right)+K / r \\
=\left(b_{n}+K / r\right) \cdot \prod_{s=n}^{t-1}\left(1+r+\left(C_{s}-r\right) \cdot h_{s}\left(B_{s}\left(b_{n}, F(n, N ; \bar{D}) ; \bar{D}\right)\right)\right) \\
\quad(t=n+1, n+2, \cdots, N+1),
\end{gathered}
$$

applying (5.27) successively.

Now, we have the following

THEOREM 5.4. Let $\widetilde{\Im}(0, \infty)$ be the set of all strategic functions $F(0, \infty)$ 's which 
satisfy the relation (5.17) on the domain $\bar{D}$. Then, we have

$$
\widetilde{\mho}(0, \infty)=\mathfrak{F}(0, \infty ; \bar{D}) .
$$

Proof. We have $\widetilde{\mho}(0, \infty) \subseteq \mathfrak{F}(0, \infty ; \bar{D})$ from Theorem 5.3. Let us verify the relation $\widetilde{\mho}(0, \infty) \supseteqq \mathfrak{F}(0, \infty ; \bar{D})$.

First, let a strategic function $\hat{F}(0, \infty) \equiv\left\{\hat{f}_{0}\left(e_{0}\right), \hat{f}_{1}\left(e_{1}\right), \hat{f}_{2}\left(e_{2}\right), \cdots\right\}$ be a strategic function which does not belong to $\widetilde{f}(0, \infty)$. Then, there is a time $t$ at which (i) the relation $0 \lesssim F(0, t-1 ; \hat{F}(0, \infty)) \lesssim(1+r) \cdot(e+K / r) /(\beta+r)$ holds true on the domain $\bar{D}$, and (ii) we can choose such a number $\hat{b}_{t}$ in the domain $\bar{D}$ as the relation

$$
\hat{f}_{t}\left(\hat{b}_{t}\right)>(1+r) \cdot\left(\hat{b}_{t}+K / r\right) /(\beta+r)
$$

holds true.

Now, we have the following

LEMMA 5.4.1. In S. D. M.S. $-I$, if $\hat{b}_{t} \in \bar{D}$ and $\hat{f}_{t}\left(\hat{b}_{t}\right)>(1+r) \cdot\left(\hat{b}_{t}+K / r\right) /(\beta+r)$, we can choose such a positive number $\delta_{1}$ and two numbers $b^{\prime}$ and $b^{\prime \prime}$ as $-K / r \leqq b^{\prime} \leqq \hat{b}_{t}<b^{\prime \prime}$ and the relation

$$
P\left\{B_{t+1}<-K / r \mid b^{\prime} \leqq b_{t}<b^{\prime \prime}, \hat{f}_{t}\left(e_{t}\right)\right\}>\delta_{1}>0
$$

holds true with respect to any $b_{t}$ in the domain $\left[b^{\prime}, b^{\prime \prime}\right)$, (see Fig. 5.2).

PROOF OF LEMMA 5.4.1.

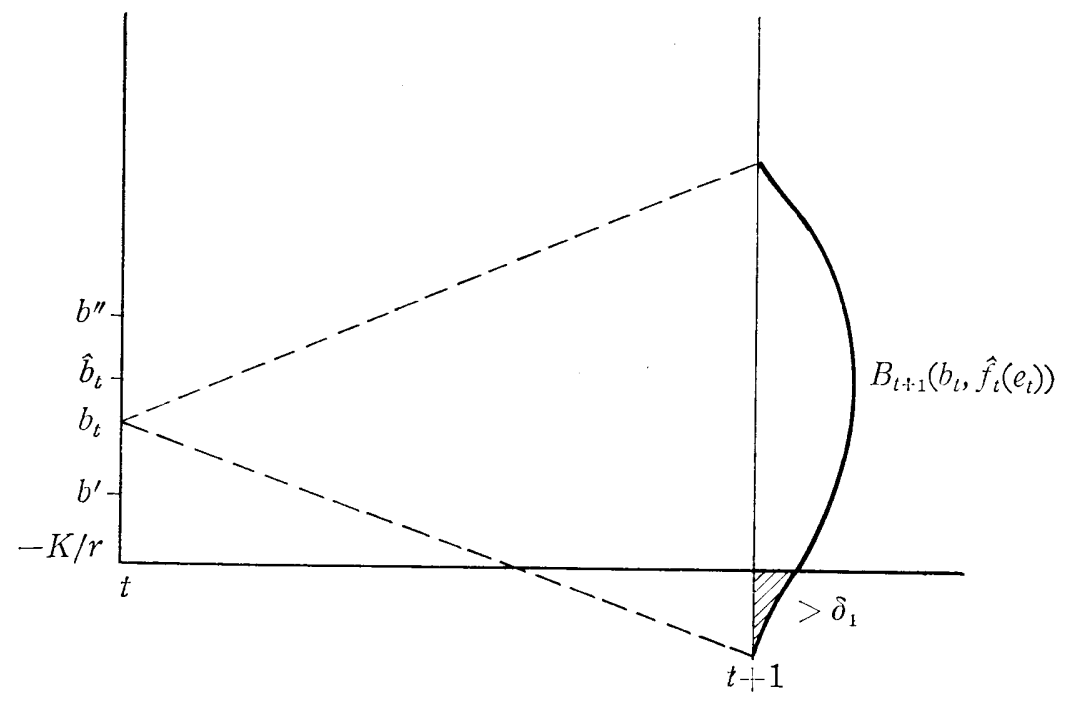

Fig. 5.2.

The function $\hat{f}_{t}\left(e_{t}\right)$ is a continuous function on the domain $\bar{D}$ except finite points, due to Condition 3.2-(ii). Therefore, if the assumption of this lemma is true, we can choose a positive number $\varepsilon$ and two numbers $b^{\prime}$ and $b^{\prime \prime}$ so that (i) $-K / r \leqq b^{\prime} \leqq \hat{b}_{t}<b^{\prime \prime}$ may hold, and (ii) the function $\hat{f}_{t}\left(e_{t}\right)$ may be continuous in the domain $\left[b^{\prime}, b^{\prime \prime}\right)$, and (iii) the relation

$$
\hat{f}_{t}\left(b_{t}\right)>(1+r) \cdot\left(b_{t}+K / r\right) /(\beta+r-\varepsilon) \geqq 0
$$


may hold true with respect to any $b_{\iota}$ in the domain $\left[b^{\prime}, b^{\prime \prime}\right)$, (see Fig. 5.3). Hence, we have

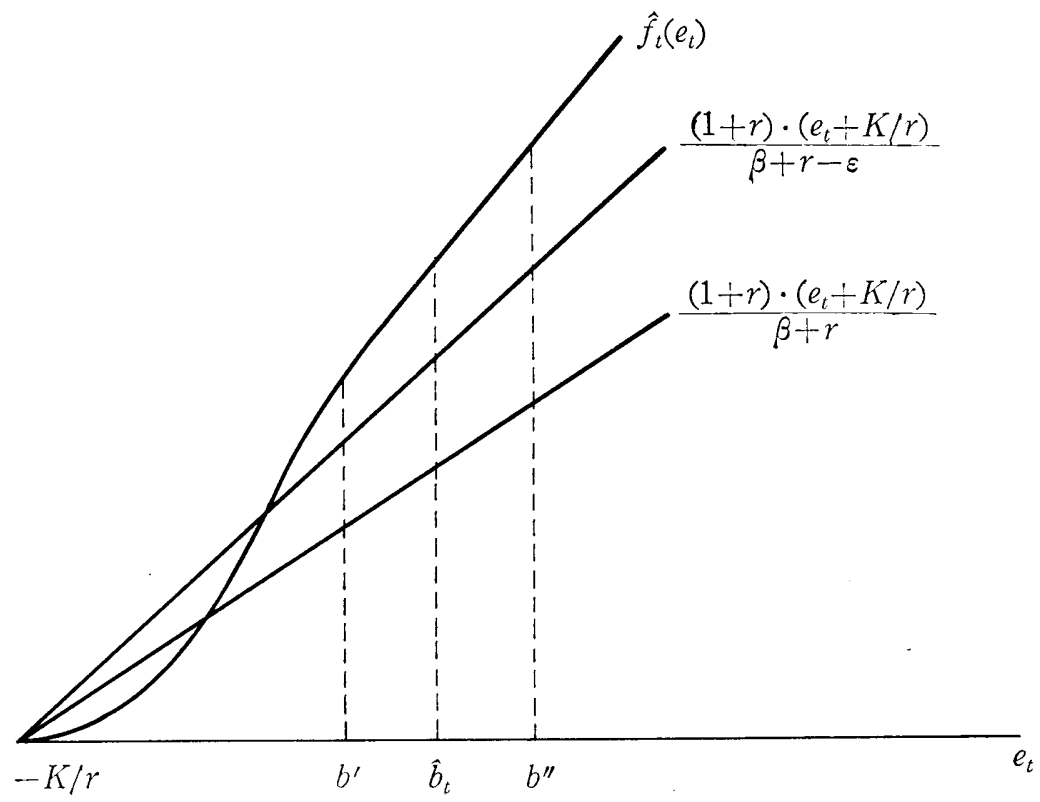

Fig. 5.3.

$$
(1+r) \cdot\left(b_{t}+K / r\right)+(-\beta-r+\varepsilon) \cdot \hat{f}_{t}\left(b_{t}\right)<0
$$

with respect to any $b_{t}$ in the domain $\left[b^{\prime}, b^{\prime \prime}\right)$.

Furthermore, if we assign the positive number $\varepsilon$ employed in (5.33), there is a positive number $\delta_{1}$ such that the relation $P\left\{C_{t}<-\beta+\varepsilon\right\}>\delta_{1}>0$ holds true, due to

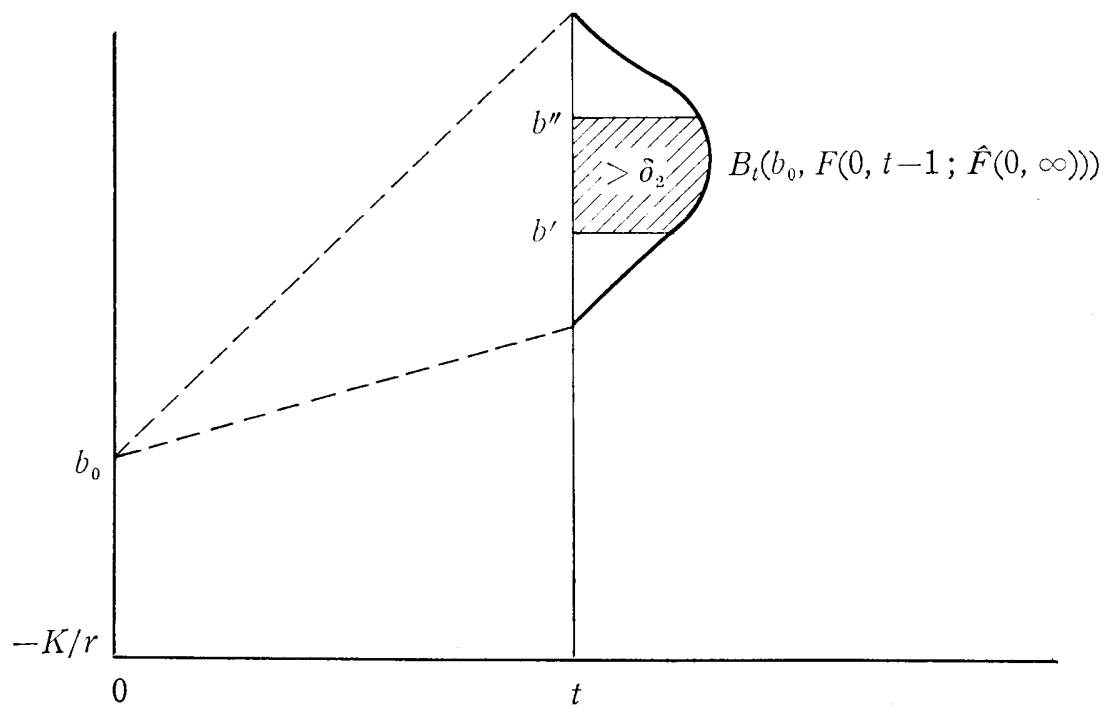

Fig. 5.4. 
Condition 3.1-(ii). Therefore, we have

$$
\begin{aligned}
P\{(1+r) & \cdot\left(b_{t}+K / r\right)+\left(C_{t}-r\right) \cdot \hat{f}_{t}\left(b_{t}\right) \\
& \left.\leqq(1+r) \cdot\left(b_{t}+K / r\right)+(-\beta-r+\varepsilon) \cdot \hat{f}_{t}\left(b_{t}\right)\right\}>\delta_{1}>0
\end{aligned}
$$

with respect to any $b_{t}$ in the domain $\bar{D}$ (and also in the domain $\left[b^{\prime}, b^{\prime \prime}\right)$ ).

Connecting (5.33) and (5.34) with (5.6), we have (5.31). LEMMA 5.4.1. Q. E. D.

LEMMA 5.4.2. If we assign any two numbers $b^{\prime}$ and $b^{\prime \prime}\left(b^{\prime}<b^{\prime \prime}\right)$ in the domain $\bar{D}$, we can choose such a positive number $\delta_{2}$ and a number $b_{0}$ as the relation

$$
P\left\{b^{\prime} \leqq B_{t}<b^{\prime \prime} \mid b_{0}, F(0, t-1 ; \hat{F}(0, \infty))\right\}>\delta_{2}>0
$$

holds true, (see Fig. 5.4).

Proof of Lemma 5.4.2. Applying the notation (5.24) to the strategic function $F(0, t-1 ; \hat{F}(0, \infty))$, let us denote as follows :

$$
\begin{aligned}
& \hat{f}_{s}\left(e_{s}\right) \equiv\left(e_{s}+K / r\right) \cdot \hat{h}_{s}\left(e_{s}\right), \quad(s=0,1,2, \cdots, t-1) \\
& \hat{H}(0, t-1) \equiv\left\{\hat{h}_{0}\left(e_{0}\right), \hat{h}_{1}\left(e_{1}\right), \cdots, \hat{h}_{t-1}\left(e_{t-1}\right)\right\} .
\end{aligned}
$$

Now, since the assumption (5.25) is employed, we have

$$
P\left\{\bigcap_{1 \leqq s \leqq t}\left(B_{s}+K / r \geqq 0\right) \mid b_{0}+K / r \geqq 0,0 \lesssim F(0, t-1 ; \hat{F}(0, \infty)) \lesssim(1+r) \cdot(e+K / r) /(\beta+r)\right\}
$$

$$
=P\left\{\bigcap_{1 \leqq s \geqq t}\left(B_{s}+K / r \geqq 0\right) \mid b_{0}+K / r \geqq 0,0 \lesssim \hat{H}(0, t-1) \lesssim(1+r) /(\beta+r)\right\}=1,
$$

according to the definition of the functions $F(0, t-1 ; \hat{F}(0, \infty))$ and $\hat{H}(0, t-1)$. Therefore, if we apply the functions $F(n, N ; \hat{F}(0, \infty))$ and $\hat{H}(0, t-1)$ in (5.28), we have

$$
\begin{gathered}
B_{s}\left(b_{0}, \hat{H}(0, t-1)\right)+K / r=\left(b_{0}+K / r\right) \cdot \prod_{i=0}^{s-1}\left(1+r+\left(C_{i}-r\right) \cdot \hat{h}_{i}\left(B_{i}\left(b_{0}, \hat{H}(0, t-1)\right)\right)\right. \\
\left(s=1,2, \cdots, t ; B_{0}=b_{0}\right) .
\end{gathered}
$$

Hence, we can see that if a vector $C=\left\{c_{0}, c_{1}, \cdots, c_{t-1}\right\}$ is assigned, $e_{t}$ is determined by $e_{0}$ through the following recurring relation

$$
e_{s}+K / r=\left(e_{0}+K / r\right) \cdot \prod_{i=0}^{s-1}\left(1+r+\left(c_{i}-r\right) \cdot \hat{h}_{i}\left(e_{i}\right)\right) \quad(s=1,2, \cdots, t),
$$

and $e_{t}$ can be recognized as a function of $e_{0}$ on the domain $\bar{D}$. For the sake of simplicity, let us denote the function of $e_{0}$ as

$$
e_{t}=\zeta_{t}\left(e_{0} ; \boldsymbol{C}, \hat{H}(0, t-1)\right) \text {. }
$$

Then, we have the following:

LEMMA 5.4.2.-1. If $-\beta<\boldsymbol{C}<\gamma$, the function $e_{t}=\zeta_{t}\left(e_{0} ; \boldsymbol{C}, \hat{H}(0, t-1)\right)$ is a continuous function of $e_{0}$ on the domain $\bar{D}$.

PROOF OF LEMMA 5.4.2-1. According to (5.39), we have a function of $e_{0}$,

$$
e_{1}=\left(e_{0}+K / r\right) \cdot\left(1+r+\left(c_{0}-r\right) \cdot \hat{h}_{0}\left(e_{0}\right)\right)-K / r \quad(\in \bar{D}),
$$

which is continuous on the domain $\bar{D}$, since we have $-\beta<c_{0}<\gamma$ and $0 \leqq \hat{h}_{0}\left(e_{0}\right)$ $\leqq(1+r) /(\beta+r)$ on that domain. 
Next, let us assume that (i) the function $e_{s}$ defined by (5.40) is a continuous function of $e_{0}$ on the domain $\bar{D}$, and (ii) the domain of $e_{s}$ is also $\bar{D}$. Then, according to (5.39), we have

$$
e_{s+1}=\left(e_{s}+K / r\right) \cdot\left(1+r+\left(c_{s}-r\right) \cdot \hat{h}_{s}\left(e_{s}\right)\right)-K / r .
$$

We can admit that (i) $e_{s+1}$ is a continuous function of $e_{s}$ on the domain $\bar{D}$ and also it is a continuous function of $e_{0}$ on the domain $\bar{D}$, and (ii) the domain of $e_{s+1}$ is $\bar{D}$.

Applying the mathematical induction, we can admit this lemma.

LEMMA 5.4.2-1. Q.E.D.

Next, let us employ the following

Definition 5.3. Let $\left\{C ; b_{0}, b_{t}, \hat{H}(0, t-1)\right\}$ be a set of all vectors $C$ 's so that the relation $\zeta_{t}\left(b_{0} ; C, \hat{H}(0, t-1)\right)=b_{t}$ holds true for assigned $b_{0}, b_{t}$ and $\hat{H}(0, t-1)$. And let $\left\{\boldsymbol{C} ; b_{0}, D, \hat{H}(0, t-1)\right\}$ be a set $\bigcup_{b_{t}=D}\left\{\boldsymbol{C} ; b_{0}, b_{t}, \hat{H}(0, t-1)\right\}$ defined for assigned $b_{0}$, (a domain) $D$ and (the fnnction) $\hat{H}(0, t-1)$. That is, we may also define as follows.

$$
\begin{aligned}
& \left\{\boldsymbol{C} ; b_{0}, b_{t}, \hat{H}(0, t-1)\right\}=\left\{\boldsymbol{C} ; \boldsymbol{\zeta}_{t}\left(b_{0} ; \boldsymbol{C}, \hat{H}(0, t-1)\right)=b_{t}\right\}, \\
& \left\{\boldsymbol{C} ; b_{0}, D, \hat{H}(0, t-1)\right\}=\left\{\boldsymbol{C} ; \boldsymbol{\zeta}_{t}\left(b_{0} ; \boldsymbol{C}, \hat{H}(0, t-1)\right) \in D\right\} .
\end{aligned}
$$

Then, we have the following

LEMMA 5.4.2-2. Let $\Re$ be the set of all rational numbers which are not smaller than $-K / r$. If $D_{0}$ is an open sub-set of $\bar{D}$, then we have

$$
\bigcup_{\boldsymbol{b} \in \boldsymbol{\Re}}\left\{\boldsymbol{C} ; b, D_{0}, \hat{H}(0, t-1)\right\}=\bigcup_{b \in \overline{\boldsymbol{D}}}\left\{\boldsymbol{C} ; b, D_{0}, \hat{H}(0, t-1)\right\} \text {. }
$$

Proof of Lemma 5.4.2-2. It is easy to see that the following relation is true.

$$
\bigcup_{b \in \Re}\left\{\boldsymbol{C} ; b, D_{0}, \hat{H}(0, t-1)\right\} \subseteq \underset{b=\bar{D}}{\bigcup}\left\{\boldsymbol{C} ; b, D_{0}, \hat{H}(0, t-1)\right\} .
$$

Therefore, let us verify the following relation.

$$
\bigcup_{b \in \Re}\left\{\boldsymbol{C} ; b, D_{0}, \hat{H}(0, t-1)\right\} \supseteqq \underset{\boldsymbol{b}=\bar{D}}{\bigcup}\left\{\boldsymbol{C} ; b, D_{0}, \hat{H}(0, t-1)\right\} .
$$

Let $b_{0}$ be any element of $\bar{D}$, and let $\hat{C}$ be any assigned element of the set $\left\{\boldsymbol{C} ; b_{0}, D_{0}, \hat{H}(0, t-1)\right\}$. Then, according to Definition 5.3 , we have

$$
\zeta_{t}\left(b_{0} ; \hat{\boldsymbol{C}}, \hat{H}(0, t-1)\right)=b_{t} \in D_{0} .
$$

Since $D_{0}$ is an open set, we can choose such a positive number $\varepsilon$ as may cause any $e_{t}$ in the domain $\left(b_{t}-\varepsilon, b_{t}+\varepsilon\right)$ to be an element of $D_{0}$ (see Fig. 5.5).

Furthermore, since the function $e_{t}=\zeta_{t}\left(e_{0} ; \hat{\boldsymbol{C}}, \hat{H}(0, t-1)\right)$ is a continuous function of $e_{0}$ on the domain $\bar{D}$ from Lemma $5.4 .2-1$, we can choose a positive number $\delta$ so that the relation

$$
\left|\zeta_{t}\left(b_{0} ; \hat{\boldsymbol{C}}, \hat{H}(0, t-1)\right)-\zeta_{t}\left(e_{0} ; \hat{\boldsymbol{C}}, \hat{H}(0, t-1)\right)\right|=\left|b_{t}-e_{t}\right|<\varepsilon
$$

holds true on the domain $\bar{D} \cap\left\{e_{0} ;\left|b_{0}-e_{0}\right|<\delta\right\}$. We can choose a rational number $R$ in the set $\bar{D} \cap\left\{e_{0} ;\left|b_{0}-e_{0}\right|<\delta\right\}$, and we have $\zeta_{t}(R ; \hat{C}, \hat{H}(0, t-1)) \in D_{0}$, (see Fig. 5.5).

That is, we have 


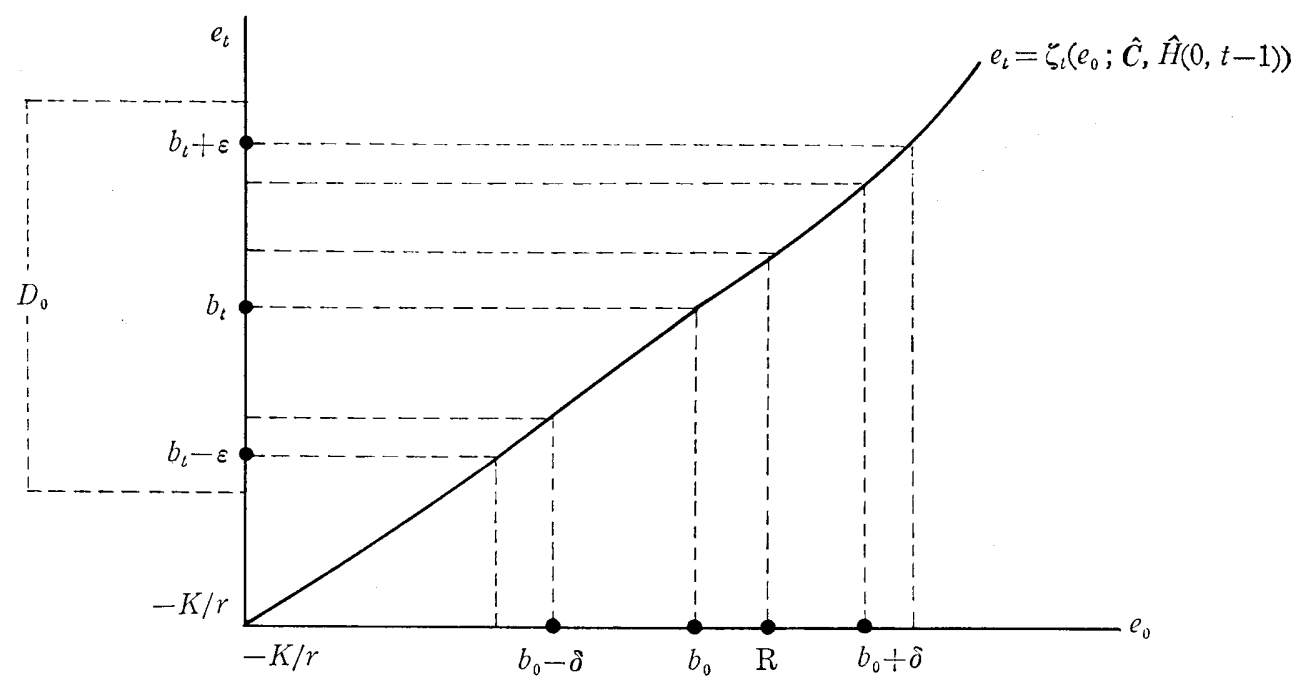

Fig. 5.5.

$$
\bigcup_{b \in \Re}\left\{\boldsymbol{C} ; b, D_{0}, \hat{H}(0, t-1)\right\} \supseteqq\left\{\boldsymbol{C} ; b_{0}, D_{0}, \hat{H}(0, t-1)\right\}
$$

with respect to any assigned $b_{0}$ in the domain $\bar{D}$. Hence we have also

$$
\bigcup_{b \in \Re}\left\{\boldsymbol{C} ; b, D_{0}, \hat{H}(0, t-1)\right\} \supseteq \bigcup_{b \in \bar{D}}\left\{\boldsymbol{C} ; b, D_{0}, \hat{H}(0, t-1)\right\} .
$$

According to (5.45) and (5.50), we can admit this lemma. LEMMA 5.4.2-2. Q. E. D.

LEMMA 5.4.2-3. Let $b^{\prime}, b^{\prime \prime}, \tilde{b}^{\prime}$ and $\tilde{b}^{\prime \prime}$ be any four numbers such that $-K / r \leqq b^{\prime}<b^{\prime \prime}$ $\leqq \tilde{b}^{\prime}<\tilde{b}^{\prime \prime}$. And let $D_{0}$ and $D_{1}$ be two domains $\left(b^{\prime}, b^{\prime \prime}\right)$ and $\left[\tilde{b}^{\prime}, \tilde{b}^{\prime \prime}\right)$, respectively. Then, we have

$$
\left\{\boldsymbol{C} ; b_{0}^{\prime}, D_{1}, \hat{H}(0, t-1)\right\} \cong \bigcup_{b \in \Re \cap\left[-K^{\prime}, b_{0}^{\prime}\right]}\left\{\boldsymbol{C} ; b, D_{0}, \hat{H}(0, t-1)\right\}
$$

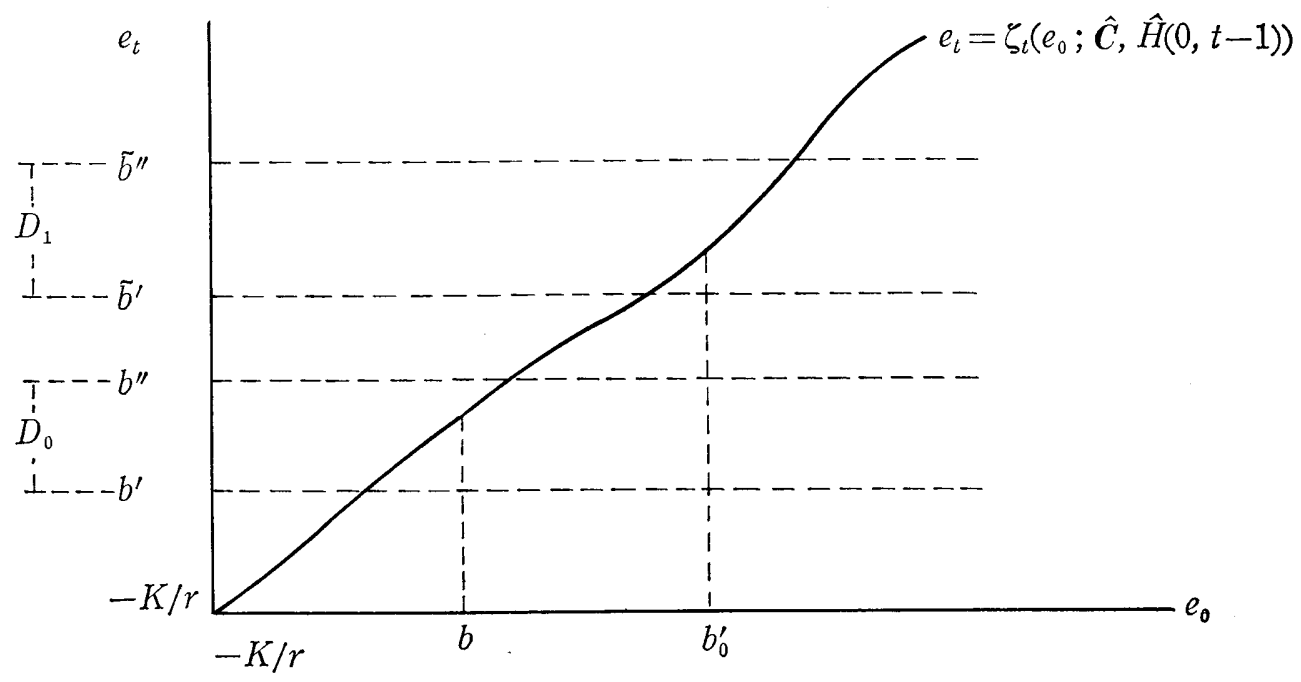

Fig. 5.6. 
with respect to any $b_{0}^{\prime}$ in the domain $\bar{D}$, (see Fig. 5.6).

Proof of Lemma 5.4.2-3. Let $\hat{C}$ be any element of the set $\left\{C ; b_{0}^{\prime}, D_{1}, \hat{H}(0, t-1)\right\}$, then we have $\zeta_{t}\left(b_{0}^{\prime} ; \hat{\boldsymbol{C}}, \hat{H}(0, t-1)\right) \in D_{1}$ according to Definition 5.3.

Now, since (i) we have $\zeta_{t}(-K / r ; \hat{\boldsymbol{C}}, \hat{H}(0, t-1))=-K / r$ due to the definition of the function $\zeta_{t}$ defined by (5.40) in Combination with (5.39), and (ii) the function $\zeta_{t}\left(e_{0} ; \hat{\boldsymbol{C}}, \hat{H}(0, t-1)\right)$ is a continuous function of $e_{0}$ on the domain $\bar{D}$ according to Lemma 5.4.2-1, then we can choose a number $b$ in the domain $\left[-K / r, b_{0}^{\prime}\right]$ so that $\zeta_{t}(b$; $\hat{\boldsymbol{C}}, \hat{H}(0, t-1)) \in D_{0}$ holds true, (see Fig. 5.6).

Therefore, we have

$$
\left\{\boldsymbol{C} ; b_{0}^{\prime}, D_{1}, \hat{H}(0, t-1)\right\} \cong \underset{b \equiv \bar{D} \cap\left[-K \mid r, b_{0}^{\prime}\right]}{\bigcup}\left\{\boldsymbol{C} ; b, D_{0}, \hat{H}(0, t-1)\right\} .
$$

Due to this relation (5.52) and Lemma 5.4.2-2, we can admit the relation (5.51), since the domain $D_{0}$ is an open interval in the domain $\bar{D}$. LEMma 5.4.2-3. Q. E. D.

Next, let $m\left\{\boldsymbol{C} ; b_{0}, D, \hat{H}(0, t-1)\right\}$ be the probability measure of a set $\{\boldsymbol{C}$; $\left.b_{0}, D, \hat{H}(0, t-1)\right\}$, then, the probability measure is equal to $P\left\{B_{t}\left(b_{0}, \hat{H}(0, t-1)\right) \in D\right\}$. If we put a domain $\left(b^{\prime}, b^{\prime \prime}\right)(\subset \bar{D})$ as $D_{0}$, we have

$$
m\left\{C ; b_{0}, D_{0}, \hat{H}(0, t-1)\right\}=P\left\{b^{\prime}<B_{t}<b^{\prime \prime} \mid b_{0}, \hat{H}(0, t-1)\right\}
$$

hence, we have the following:

LEMMA 5.4.2-4. Let $b^{\prime}, b^{\prime \prime}, \tilde{b}^{\prime}$ and $\tilde{b}^{\prime \prime}$ be any four numbers in the domain $\bar{D}$ so that $b^{\prime}<b^{\prime \prime} \leqq \tilde{b}^{\prime}<\tilde{b}^{\prime \prime}$, and let $D_{0}$ and $D_{1}$ may be two domains $\left(b^{\prime}, b^{\prime \prime}\right)$ and $\left[\tilde{b}^{\prime}, \tilde{b}^{\prime \prime}\right)$, respectively. If there is a number $b_{0}^{\prime}$, in the domain $\bar{D}$ so that

$$
m\left\{C ; b_{0}^{\prime}, D_{1}, \hat{H}(0, t-1)\right\}>0,
$$

there is also a rational number $b_{0}$ so that $-K / r \leqq b_{0} \leqq b_{0}^{\prime}$ and

$$
m\left\{\boldsymbol{C} ; b_{0}, D_{0}, \hat{H}(0, t-1)\right\}>0
$$

holds true, (see Fig. 5.7).

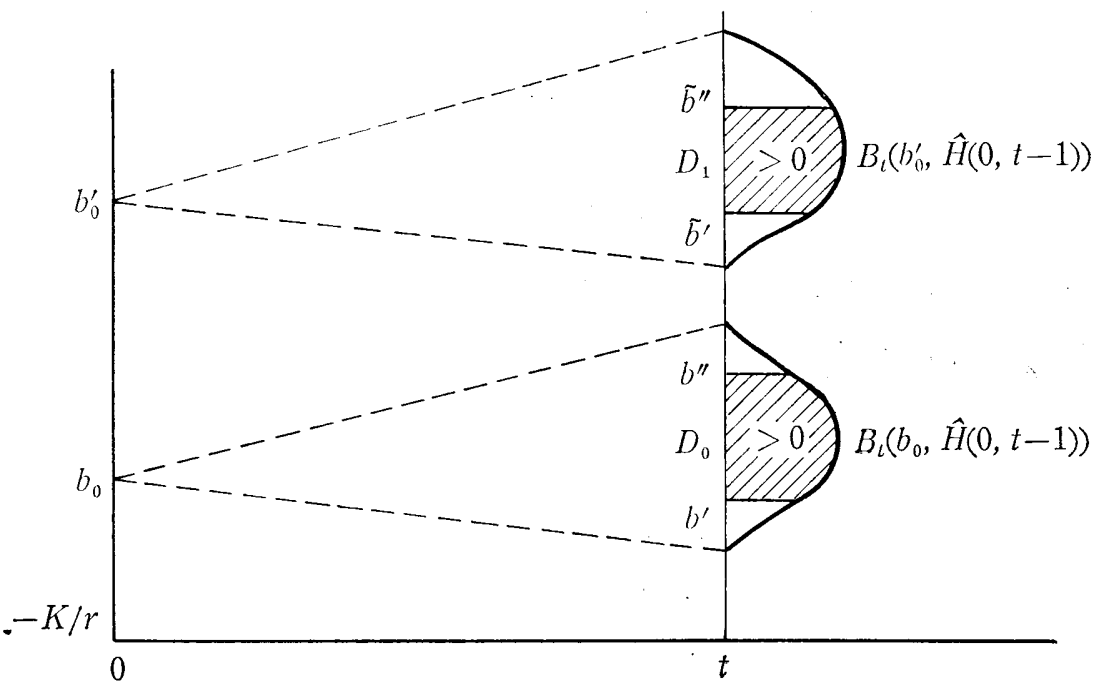

Fig. 5.7. 
Proof of Lemma 5.4.2-4.

According to Lemma 5.4.2-3 and the relation (5.54), we have a relation

$$
0<m\left\{\boldsymbol{C} ; b_{0}^{\prime}, D_{1}, \hat{H}(0, t-1)\right\} \leqq m\left[\bigcup_{b \in \Re \cap\left[-K / r, b_{0}^{\prime}\right]}\left\{\boldsymbol{C} ; b, D_{0}, \hat{H}(0, t-1)\right\}\right] .
$$

Since the set of rational numbers $\Re \cap\left[-K / r, b_{0}^{\prime}\right]$ is a denumerable set, let us denote the set by an infinite sequence of rational numbers $\left\{R_{1}, R_{2}, R_{3}, \cdots\right\}$. Then, we have

$$
0<m\left[\bigcup_{b \in \Re \cap\left[-K / r, b_{0}^{\prime}\right]}\left\{\boldsymbol{C} ; b, D_{0}, \hat{H}(0, t-1)\right\}\right] \leqq \sum_{i=1}^{\infty} m\left\{\boldsymbol{C} ; R_{i}, D_{0}, \hat{H}(0, t-1)\right\} .
$$

That is, we can choose, at least, a rational number $R_{i}$ so that $m\left\{C ; R_{i}, D_{0}, \hat{H}(0, t-1)\right\}$ $>0$. Putting as $b_{0}=R_{i}$, we can admit this lemma.

LEMMA 5.4.2-4. Q. E. D.

LEMMA 5.4.2-5. If $\tilde{b}^{\prime}$ is any assigned number so that $\tilde{b}^{\prime}>-K / r$, there are two numbers $b_{0}^{\prime}(>-K / r)$ and $\tilde{b}^{\prime \prime}\left(>\tilde{b}^{\prime}\right)$ so that the following relation holds true, (see Fig. 5.8).

$$
P\left\{\tilde{b}^{\prime} \leqq B_{t}<\tilde{b}^{\prime \prime} \mid b_{0}^{\prime}, \hat{H}(0, t-1)\right\}>0 .
$$

Proof of Lemma 5.4.2-5.

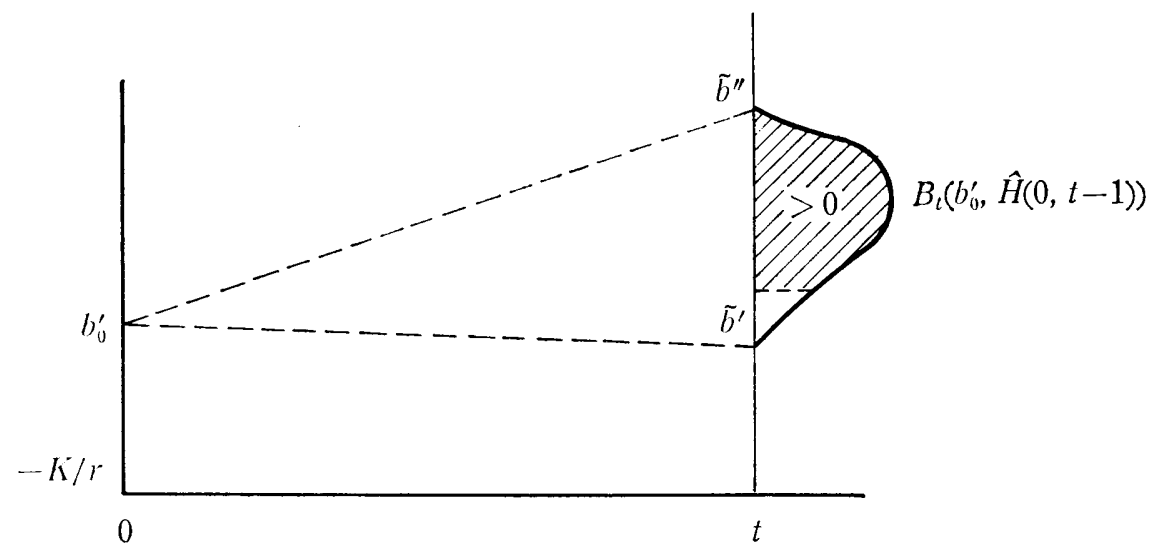

Fig. 5.8.

We have the relation

$$
P\left\{\bigcap_{0 \leqq s \leqq t-1}\left(B_{s} \geqq-K / r\right) \mid b_{0} \in \bar{D}, \hat{H}(0, t-1)\right\}=1
$$

according to Theorem 5.3 and the definition of the function $\hat{H}(0, t-1)$. Therefore, we have also

$$
P\left\{\bigcap_{0 \leqq s \leqq t-1}\left(0 \leqq \hat{h}_{s}\left(B_{s}\right) \leqq(1+r) /(\beta+r)\right) \mid b_{0} \in \bar{D}, \hat{H}(0, t-1)\right\}=1 .
$$

Hence, we have

$$
\begin{gathered}
P\left\{1+r \leqq 1+r+\left(C_{s}-r\right) \cdot \hat{h}_{s}\left(B_{s}\right) \mid b_{0} \geqq-K / r, \hat{H}(0, t-1)\right\} \\
=P\left\{0 \leqq\left(C_{s}-r\right) \cdot \hat{h}_{s}\left(B_{s}\right) \mid b_{0} \geqq-K / r, \hat{H}(0, t-1)\right\} \\
\geqq P\left\{C_{s}-r>0\right\} \cdot P\left\{0 \leqq \hat{h}_{s}\left(B_{s}\right) \mid b_{s} \geqq-K / r, \hat{H}(0, t-1)\right\}=\mu \\
(s=0,1,2, \cdots, t-1),
\end{gathered}
$$


from (5.60) in combination with Conditions 3.1-(ii) and 3.2-(i). Applying (5.61) to the relation (5.38), we have

$$
P\left\{\left(b_{0}+K / r\right) \cdot(1+r)^{t} \leqq B_{t}+K / r \mid b_{0} \geqq-K / r, \hat{H}(0, t-1)\right\} \geqq \mu^{\iota}>0 .
$$

If we choose a number $b_{0}^{\prime}(>-K / r)$ so that $\left(b_{0}^{\prime}+K / r\right) \cdot(1+r)^{t}=\tilde{b}^{\prime}+K / r$, and a sufficiently large number $\tilde{b}^{\prime \prime}$, then we have

$$
P\left\{\tilde{b}^{\prime}+K / r \leqq B_{t}+K / r \leqq \tilde{b}^{\prime \prime}+K / r\right\}=P\left\{\tilde{b}^{\prime} \leqq B_{t} \leqq \tilde{b}^{\prime \prime} \mid b_{0}^{\prime}, \hat{H}(0, t-1)\right\}>0
$$

which verifies this lemma is true.

LEMMA 5.4.2-5. Q. E. D.

Now, let $b^{\prime}$ and $b^{\prime \prime}$ be any two numbers so that $-K / r \leqq b^{\prime}<b^{\prime \prime}$. If we choose a number $\tilde{b}^{\prime}$ larger than $b^{\prime \prime}$, and choose a sufficiently large number $\tilde{b}^{\prime \prime}$, then we can choose a number $b_{0}^{\prime}(>-K / r)$ so that (5.58) holds true, according to Lemma 5.4.2-5. Therefore, we can choose a rational number $b_{0}$ and a positive number $\delta_{2}$ so that the relation $-K / r<b_{0}<b_{0}^{\prime}$ and the relation

$$
\begin{aligned}
P\left\{b^{\prime} \leqq\right. & B_{t}<b^{\prime \prime} \mid b_{0}, F(0, t-1 ; \hat{F}(0, \infty)\} \\
& \geqq P\left\{b^{\prime}<B_{t}<b^{\prime \prime} \mid b_{0}, \hat{H}(0, t-1)\right\}>\delta_{2}>0
\end{aligned}
$$

hold true, according to Lemma 5.4.2-4, (see Fig. 5.9).

Lemma 5.4.2. Q.E. D.

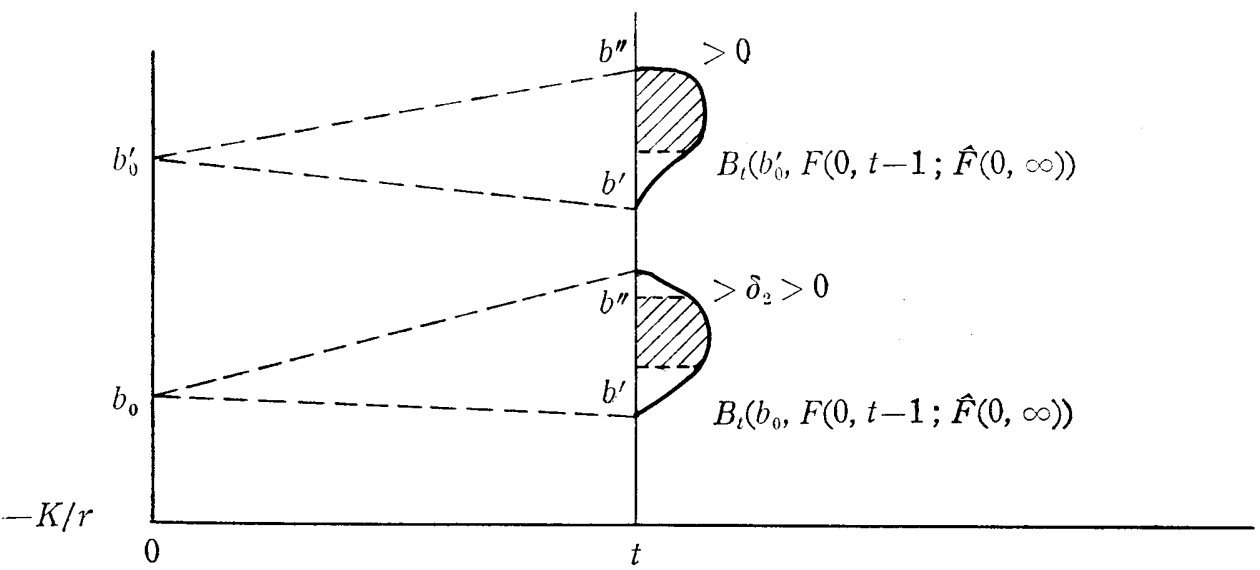

Fig. 5.9.

Lemmas 5.4.1 and 5.4.2 show that we can choose a number $b_{0}$ in the domain $\bar{D}$ and two positive numbers $\delta_{1}$ and $\delta_{2}$ so that the relation

$$
\begin{aligned}
P\left\{B_{t+1}<-\right. & \left.K / r \mid b_{0}, \hat{F}(0, \infty)\right\} \\
\geqq & \min _{b^{\prime} \leqq b_{t} \leqq b^{\prime \prime}} P\left\{B_{t+1}<-K / r \mid b_{t}, \hat{f}_{t}\left(e_{t}\right)\right\} \\
& \quad \cdot P\left\{b^{\prime} \leqq B_{t}<b^{\prime \prime} \mid b_{0}, F(0, t-1 ; \hat{F}(0, \infty)\} \geqq \delta_{1} \cdot \delta_{2}\right.
\end{aligned}
$$

holds true. That is, we can admit that if $\hat{F}(0, \infty) \notin \widetilde{F}(0, \infty)$, then we have also $\hat{F}(0, \infty)$ $\notin \mathfrak{F}(0, \infty ; \bar{D})$, according to Corollary 5.2.2. Hence, we have the relation $\widetilde{F}(0, \infty)$ $\supseteq \mathfrak{F}(0, \infty ; \bar{D})$, and we can admit this theorem due to Theorem 5.3.

THEOREM 5.4. Q.E.D. 


\section{§6. Simplification of the Stochastic Dynamic Programming in S. D. M. S.-I}

In order to evade difficulties in derivation and calculation of the $\left(b_{0}, \xi\right)$-optimal strategic function on the basis of the relation (4.5) in S. D. M. S.-I, the sets $\widetilde{F}(n, N ; \bar{D})$ 's $(n=0,1,2, \cdots, N ; N=0,1,2, \cdots)$ will play an important role. Furthermore, let us prepare the following definitions.

Definition 6.1. In S.D. M.S.-I, if (i) a $\left(b_{n}, \xi\right)$-criterion functional $U(F(n, N ; \bar{D})$; $\left.b_{n}, \xi\right)$ is finite or negative infinite on $\mathfrak{F}(n, N ; \bar{D})$, and a strategic function $\hat{F}(n, N ; \bar{D})$ maximizes the $\left(b_{n}, \xi\right)$-criterion functional $U\left(F(n, N ; \bar{D}) ; b_{n}, \xi\right)$ in $\mathfrak{F}(n, N ; \bar{D})$, then the strategic function $\hat{F}(n, N ; \bar{D})$ is called " $\left(\bar{D} ; b_{n}, \xi\right)$-optimal."

A $\left(\bar{D} ; b_{n}, \xi\right)$-optimal strategic function is denoted by $F^{*}\left(n, N ; \bar{D} ; b_{n}, \xi\right)$, and the set of all $\left(\bar{D} ; b_{n}, \xi\right)$-optimal strategic function is denoted by $\widetilde{\mho}^{*}\left(n, N ; \bar{D} ; b_{n}, \xi\right)$.

DeFinition 6.2. In S.D.M.S.-I, if (i) $a\left(B_{n}, \xi\right)$-criterion functional $E\{U(F(n, N$; $\left.\left.\bar{D} ; B_{n}, \xi\right)\right\}$ (where $\left.D\left(B_{n}\right) \subseteq \bar{D}\right)$ is finite or negative infinite on $\mathfrak{F}(n, N ; \bar{D})$, and (ii) a strategic function $\hat{F}(n, N ; \bar{D})$ maximizes the $\left(B_{n}, \xi\right)$-criterion functional $E\{U(F(n, N$; $\left.\left.\bar{D}) ; B_{n}, \xi\right)\right\}$ in $\mathfrak{F}(n, N ; \bar{D})$, then the strategic function $\hat{F}(n, N ; \bar{D})$ is called ${ }^{n_{n}}\left(\bar{D} ; B_{n}, \xi\right)$. optimal."

In what follows, a $\left(\bar{D} ; B_{n}, \xi\right)$-optimal strategic function is denoted by $F *(n, N ; \bar{D}$; $\left.B_{n}, \xi\right)$, and the set of all $\left(\bar{D} ; B_{n}, \xi\right)$-optimal strategic functions is denoted by $\mathfrak{F}^{*}(n, N$; $\left.\bar{D} ; B_{n}, \xi\right)$.

Definition 6.3. An element of the set $\bigcap_{b_{n} \in D} \mathscr{F}^{*}\left(n, N ; \bar{D} ; b_{n}, \xi\right)$ is called " $(\bar{D} ; D, \xi)$ optimal."

In what follows, a $(\bar{D} ; D, \xi)$-optimal strategic function is denoted by $F^{*}(n, N ; \bar{D}$; $D, \xi)$ and the set $\bigcap_{b_{n}=D} \mathfrak{F}^{*}\left(n, N ; \bar{D} ; b_{n}, \xi\right)$ is also denoted by $\mathfrak{F}^{*}(n, N ; \bar{D} ; D, \xi)$.

Hence, we shall verify the following two propositions.

(A) If there is a $(\bar{D} ; \bar{D}, \xi)$-optimal strategic function $F^{*}(0, N ; \bar{D} ; \bar{D}, \xi)$, the strategic function is also a $\left(b_{0}, \xi\right)$-optimal strategic function $F^{*}\left(0, N ; b_{0}, \xi\right)$, with respect to any $b_{0}$ in the domain $\bar{D}$.

(B) There is a $(\bar{D} ; \bar{D}, \xi)$-optimal strategic function $F^{*}(0, N ; \bar{D} ; \bar{D}, \xi)$ if the utility function $\xi$ satisfies the following.

Assumption 6.1. At each time $n(n=0,1,2, \cdots, N)$, if a decision maker has any amount of capital money $b_{n}$ in the domain $\bar{D}$ and he assig $n$ a last time of programming $N(N=n, n+1, n+2, \cdots)$, then

(a) the $\left(b_{n}, \xi\right)$-criterion functional $U\left(F\left(n, N ; b_{n}\right) ; b_{n}, \xi\right)$ is finite or negative infinite on $\mathfrak{F}\left(n, N ; b_{n}\right)$, and

(b) there is a strategic function $\hat{F}\left(n, N ; b_{n}\right)$ which maximizes the $\left(b_{n}, \xi\right)$-criterion functional $U\left(F\left(n, N ; b_{n}\right) ; b_{n}, \xi\right)$ in $\mathfrak{F}\left(n, N ; b_{n}\right)$ and by which the value $U\left(\hat{F}\left(n, N ; b_{n}\right)\right.$; $\left.b_{n}, \xi\right)$ is a finite value.

Assumption 6.1 is a fundamental condition in order that we can obtain a $\left(b_{0}, \xi\right)$ optimal strategic function in S. D. M. S.-I on the basis of the stochastic relation (4.5). Therefore, if we verify the above two propositions (A) and (B), the stochastic dynamic programming in S.D.M.S.-I is completed on the theoretical part, by making only a $(\bar{D} ; \bar{D}, \xi)$-optimal strategic function $F^{*}(0, N ; \bar{D} ; \bar{D}, \xi)$. 
First, let us verify the following

THEOREM 6.1. If there is a $(\bar{D} ; \bar{D}, \xi)$-optimal strategic function $F^{*}(n, N ; \bar{D} ; \bar{D}, \xi)$ in S.D.M.S.-I, the strategic function is also $a\left(b_{n}, \xi\right)$-optimal strategic function $F^{*}\left(n, N ; b_{n}, \xi\right)$, on any $b_{n}$ in the domain $\bar{D}$, and we have

$$
U\left(F^{*}(n, N ; \bar{D} ; \bar{D}, \xi) ; b_{n}, \xi\right)=U\left(F^{*}\left(n, N ; b_{n}, \xi\right) ; b_{n}, \xi\right) .
$$

PROOF. We have the following relations

$$
\mathfrak{F}^{*}(n, N ; \bar{D} ; \bar{D}, \xi) \subseteq \mathfrak{F}^{*}\left(n, N ; \bar{D} ; b_{n}, \xi\right) \quad\left(b_{n} \in \bar{D}\right)
$$

and

$$
U\left(F^{*}(n, N ; \bar{D} ; \bar{D}, \xi) ; b_{n}, \xi\right)=U\left(F^{*}\left(n, N ; \bar{D} ; b_{n}, \xi\right) ; b_{n}, \xi\right) \quad\left(b_{n} \in \bar{D}\right),
$$

due to Definitions 6.1 and 6.3.

Furthermore, we have the following

LEMMA 6.1.1. If there is a $\left(\bar{D} ; b_{n}, \xi\right)$-optimal strategic function $F *\left(n, N ; \bar{D} ; b_{n}, \xi\right)$ in S.D.M.S.-I on $a b_{n}$ in the domain $\bar{D}$, then the strategic function is also $a\left(b_{n}, \xi\right)$ optimal strategic function $F^{*}\left(n, N ; b_{n}, \xi\right)$, that is, we have the relations

$$
\mathfrak{F}^{*}\left(n, N ; \bar{D} ; b_{n}, \xi\right) \leqq \mathfrak{F}^{*}\left(n, N ; b_{n}, \xi\right) \quad\left(b_{n} \in \bar{D}\right)
$$

and

$$
U\left(F^{*}\left(n, N ; \bar{D} ; b_{n}, \xi\right) ; b_{n}, \xi\right)=U\left(F^{*}\left(n, N ; b_{n}, \xi\right) ; b_{n}, \xi\right) \quad\left(b_{n} \in \bar{D}\right) .
$$

ProOF of Lemma 6.1.1. Let $b_{n}$ be any assigned element of $\bar{D}$, and let us choose any $\left(\bar{D} ; b_{n}, \xi\right)$-optimal strategic function $F^{*}\left(n, N ; \bar{D} ; b_{n}, \xi\right)$ and any $b_{n}$-feasible strategic function $F\left(n, N ; b_{n}\right)$. Furthermore, let $f_{t}^{*}\left(e_{t} ; \bar{D} ; b_{n}, \xi\right)$ and $f_{t}\left(e_{t} ; b_{n}\right)(n \leqq t \leqq N)$ be component functions at the time $t$ of the strategic functions $F^{*}\left(n, N ; \bar{D} ; b_{n}, \xi\right)$ and $F\left(n, N ; b_{n}\right)$, respectively. Then, we can produce a strategic function $\hat{F}(n, N)=\left\{\hat{f}_{n}\left(e_{n}\right)\right.$, $\left.\hat{f}_{n+1}\left(e_{n+1}\right), \cdots, \hat{f}_{N}\left(e_{N}\right)\right\}$ so that

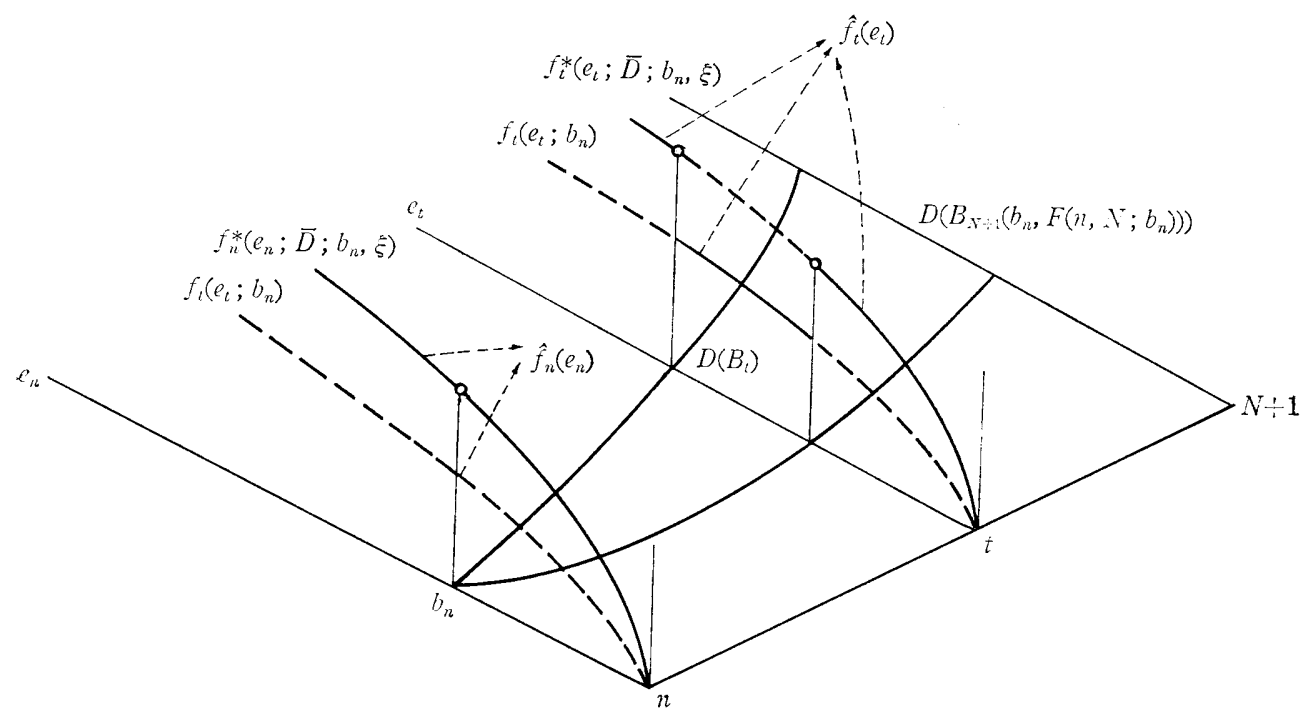

Fig. 6.1. 
(i) $\hat{f}_{t}\left(e_{t}\right)=f_{t}\left(e_{t} ; b_{n}\right)$ if $e_{t} \in D\left(B_{t}\left(b_{n}, F\left(n, N ; b_{n}\right)\right)\right)$;

(ii) $\hat{f}_{t}\left(e_{t}\right)=f_{t}^{*}\left(e_{t} ; D ; b_{n}, \xi\right)$ if $e_{t} \in D\left(B_{t}\left(b_{n}, F\left(n, N ; b_{n}\right)\right)\right)^{c} \cap \bar{D}$, $(t=n, n+1, \cdots, N), \quad$ (see Fig. 6.1).

Of course, (i) we have

$$
U\left(\hat{F}(n, N) ; b_{n}, \xi\right)=U\left(F\left(n, N ; b_{n}\right) ; b_{n}, \xi\right)
$$

according to the definition of the strategic function $\hat{F}(n, N)$, and (ii) we have

$$
U\left(F^{*}\left(n, N ; \bar{D} ; b_{n}, \xi\right) ; b_{n}, \xi\right) \geqq U\left(\hat{F}(n, N) ; b_{n}, \xi\right)
$$

since the strategic function $\hat{F}(n, N)$ is also $\bar{D}$-feasible. Hence we have

$$
U\left(F *\left(n, N ; \bar{D} ; b_{n}, \xi\right) ; b_{n}, \xi\right) \geqq U\left(F\left(n, N ; b_{n}\right) ; b_{n}, \xi\right)
$$

with respect to any $F\left(n, N ; b_{n}\right)$ in $\widetilde{F}\left(n, N ; b_{n}\right)$.

Since $\mathfrak{F}(n, N ; \bar{D}) \subseteq \mathfrak{F}\left(n, N ; b_{n}\right)$, we can admit this lemma due to (6.8).

LEMMA 6.1.1. Q. E. D.

Relations (6.2) through (6.5) show this theorem is true. THEOREM 6.1. Q.E.D.

Our next problem is to verify that there is a $(\bar{D} ; \bar{D}, \xi)$-optimal strategic function under Assumption 6.1. First, let us verify the following:

THEOREM 6.2. Let $B_{n}$ be a stochastic variable so that $D\left(B_{n}\right) \leqq \bar{D}$. If there is a $(\bar{D} ; \bar{D}, \xi)$-optimal strategic function $F^{*}(n, N ; \bar{D} ; \bar{D}, \xi)$, the strategic function is also $\left(\bar{D} ; B_{n}, \xi\right)$-optimal in S.D.M.S.-I, and we have

$$
\underset{\eta_{n}}{E}\left\{U\left(F^{*}(n, N ; \bar{D} ; \bar{D}, \xi) ; B_{n}, \xi\right)\right\}=\underset{\eta_{n}}{E}\left\{U\left(F^{*}\left(n, N ; \bar{D} ; B_{n}, \xi\right) ; B_{n}, \xi\right)\right\} .
$$

Proof. We have

$$
\begin{aligned}
\underset{\eta_{n}}{E}\left\{U\left(F^{*}(n, N ; \bar{D} ; \bar{D}, \xi) ; B_{n}, \xi\right)\right\} \\
\quad=\int_{D\left(B_{n}\right)} U\left(F^{*}(n, N ; \bar{D} ; \bar{D}, \xi) ; e_{n}, \xi\right) \cdot \eta_{n}\left(e_{n}\right) \cdot d e_{n} \\
\quad \geqq \int_{D\left(B_{n}\right)} U\left(F(n, N ; \bar{D}) ; e_{n}, \xi\right) \cdot \eta_{n}\left(e_{n}\right) \cdot d e_{n} \\
\quad=\operatorname{\vartheta }_{\boldsymbol{x}_{n}}\left\{U\left(F(n, N ; \bar{D}) ; B_{n}, \xi\right)\right\}
\end{aligned}
$$

with respect to any strategic function $F(n, N ; \bar{D})$ in $\widetilde{F}(n, N ; \bar{D})$. That is, we can admit this theorem.

Q.E.D.

Next, we have the following

THEOREM 6.3. In S.D.M.S.-I, if a utility function $\xi(e)$ satisfies Assumption 6.1, there is a $(\bar{D} ; \bar{D}, \xi)$-optimal strategic function $F^{*}(n, N ; \bar{D} ; \bar{D}, \xi)$, with respect to any assigned non-negative two integers $n$ and $N$ so that $n \leqq N$.

Proof. In order to prove this theorem, as the first step, let us verify the following

1) These strategic functions $\hat{f}_{t}\left(e_{t}\right)$ 's $(t=n, n+1, n+2, \cdots, N)$ may be discontinuous at finite points in the domain $\bar{D}$. However, such discontinuity of the strategic functions $\hat{f}_{t}\left(e_{t}\right)$ 's is admitted in S. D. M. S.-I, by Condition 3.2-(ii). 
LEMMA 6.3.1. In S.D.M.S.-I, if a utility function $\xi(e)$ satisfies Assumption 6.1, there is a $\left(\bar{D} ; b_{n}, \xi\right)$-optimal strategic function $F^{*}\left(n, N ; \bar{D} ; b_{n}, \xi\right)$, with respect to any assigned $b_{n}$ in the domain $\bar{D}$ and any assigned integers $n$ and $N(n=0,1,2, \cdots, N$; $N=0,1,2, \cdots)$.

Proof of Lemma 6.3.1. Let $F(n, N ; \bar{D})$ be any assigned $\bar{D}$-feasible strategic function and let $F^{*}\left(n, N ; b_{n}, \xi\right)$ be any $\left(b_{n}, \xi\right)$-optimal strategic function which can be chosen due to Assumption 6.1. And let $f_{t}^{*}\left(e_{t} ; b_{n}, \xi\right)(n \leqq t \leqq N)$ be component function at the time $t$ of the strategic function $F^{*}\left(n, N ; b_{n}, \xi\right)$. Then, we can make a strategic function $\hat{F}(n, N) \equiv\left\{\hat{f}_{n}\left(e_{n}\right), \hat{f}_{n+1}\left(e_{n+1}\right), \cdots, \hat{f}_{N}\left(e_{N}\right)\right\}$ so that

(i) $\hat{f}_{t}\left(e_{t}\right)=f_{t}^{*}\left(e_{t} ; b_{n}, \xi\right)$ if $e_{t} \in D\left(B_{t}\left(b_{n}, F^{*}\left(n, N ; b_{n}, \xi\right)\right)\right)$ and

(ii) $\hat{f}_{t}\left(e_{t}\right)=f_{t}\left(e_{t} ; \bar{D}\right)$ if $e_{t} \in D\left(B_{t}\left(b_{n}, F^{*}\left(n, N ; b_{n}, \xi\right)\right)\right)^{e} \cap \bar{D}, \quad(t=n, n+1, \cdots, N)$,

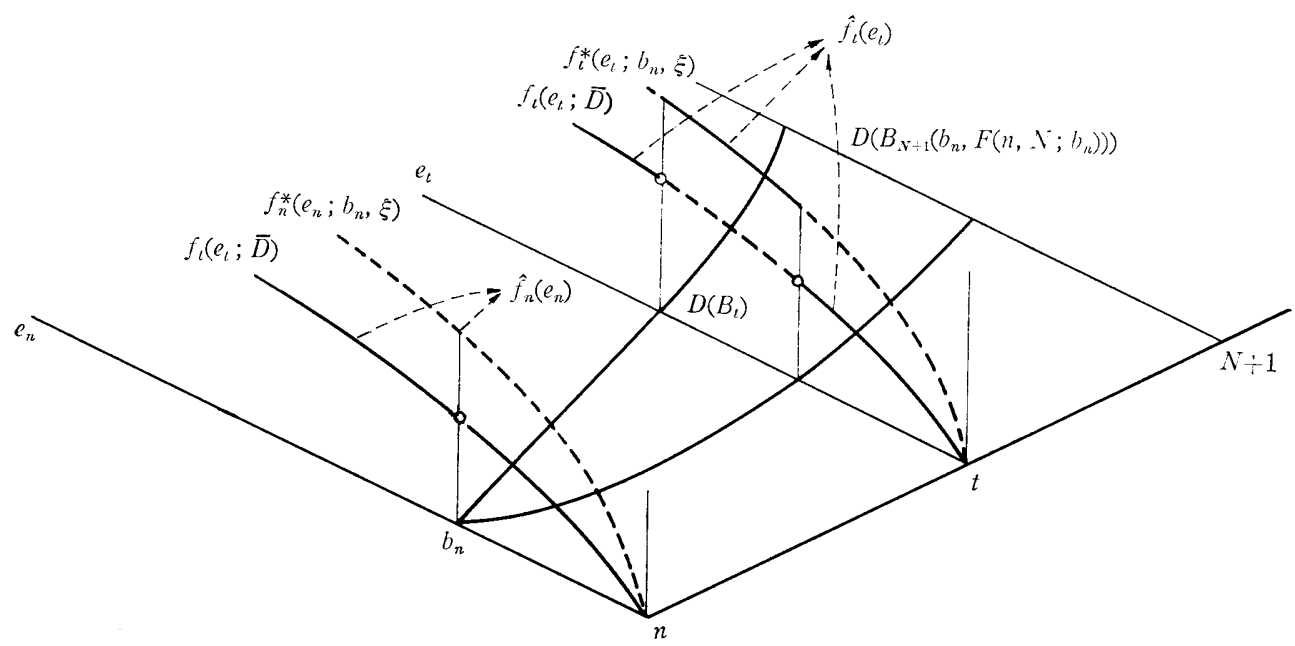

Fig. 6.2.

Of course, (i) if $b_{n} \in \bar{D}$, we have

$$
U\left(\hat{F}(n, N) ; b_{n}, \xi\right)=U\left(F^{*}\left(n, N ; b_{n}, \xi\right) ; b_{n}, \xi\right),
$$

and (ii) the strategic function $\hat{F}(n, N)$ is a $\bar{D}$-feasible strategic function due to the definition of it.

Since $\mathfrak{F}(n, N ; \bar{D}) \leqq \widetilde{F}\left(n, N ; b_{n}\right)$, the strategic function $\hat{F}(n, N)$ is a $\left(\bar{D} ; b_{n}, \xi\right)$-optimal strategic function.

LEMMA 6.3.1. Q. E. D.

Now, let $f_{s}^{*}\left(e_{s} ; \bar{D} ; b_{n}, \xi\right)$ be the component function at the time $s$ of a $\left(\bar{D} ; b_{n}, \xi\right)$ optimal strategic function $F^{*}\left(n, N ; \vec{D} ; b_{n}, \xi\right)$. And let us prove the theorem by means of the backward mathematical induction.

(A) Let us consider the case of $n=N$.

If we assign any $b_{N}$ in the domain $\bar{D}$, we can choose a $\left(\bar{D} ; b_{N}, \xi\right)$-optimal strategic function $f_{N}^{*}\left(e_{N} ; \bar{D} ; b_{N}, \xi\right)$ according to Lemma 6.3.1. Therefore, we can also choose such a strategic function $\hat{f}_{N}\left(e_{N} ; \bar{D}\right)$ as may cause the relation

$$
\hat{f}_{N}\left(b_{N} ; \bar{D}\right)=f_{N}^{*}\left(b_{N} ; \bar{D} ; b_{N}, \xi\right)
$$

to be satisfied with respect to each $b_{N}$ in the domain $\bar{D}$, (see Fig. 6.3). 


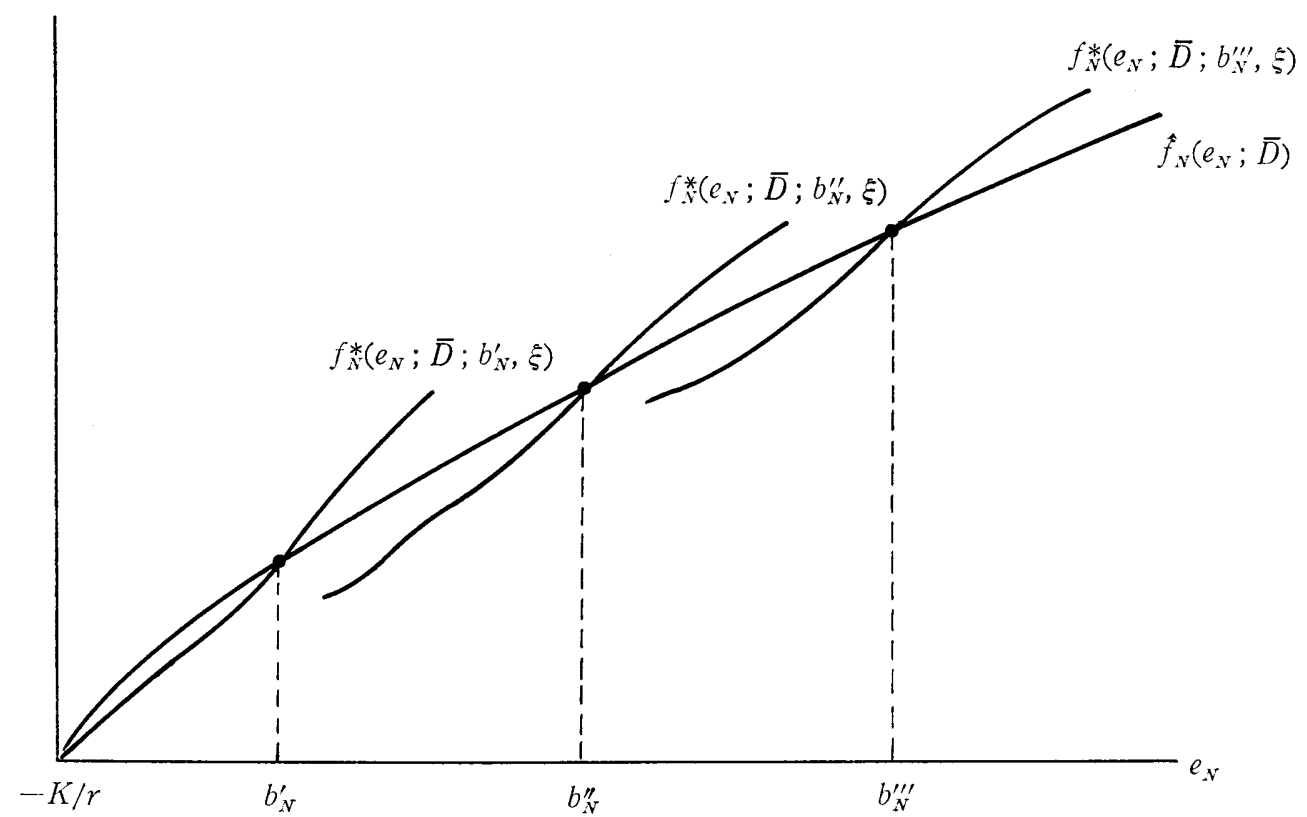

Fig. 6.3.

Then, we have

$$
\begin{aligned}
U\left(\hat{f}_{N}\left(e_{N} ; \bar{D}\right) ; b_{N}, \xi\right) & =U\left(\hat{f}_{N}\left(b_{N} ; \bar{D}\right) ; b_{N}, \xi\right) \\
& =U\left(f_{N}^{*}\left(b_{N} ; \bar{D} ; b_{N}, \xi\right) ; b_{N}, \xi\right) \\
& =U\left(f_{N}^{*}\left(e_{N} ; \bar{D} ; b_{N}, \xi\right) ; b_{N}, \xi\right),
\end{aligned}
$$

and therefore, we have

$$
\hat{f}_{N}\left(e_{N} ; \bar{D}\right) \in \mathfrak{F}^{*}\left(N, N ; \bar{D} ; b_{N}, \xi\right)
$$

with respect to any $b_{N}$ in the domain $\bar{D}$. Hence, we have

$$
\hat{f}_{N}\left(e_{N} ; \bar{D}\right) \in \bigcap_{b_{N}=D} \mathfrak{F}^{*}\left(N, N ; \bar{D} ; b_{N}, \xi\right)=\mathfrak{F}^{*}(N, N ; \bar{D} ; \bar{D}, \xi)
$$

which shows this theorem is true in the case of $n=N$.

(B) Next, let us assume (i) the utility function $\xi(e)$ satisfies Assumption 6.1, and (ii) there are $(\bar{D} ; \bar{D}, \xi)$-optimal strategic functions $F^{*}(N, N ; \bar{D} ; \bar{D}, \xi), F^{*}(N-1, N$; $\bar{D} ; \bar{D}, \xi), \cdots, F^{*}(s+1, N ; \bar{D} ; \bar{D}, \xi)$.

If we assign any $b_{s}$ in the domain $\bar{D}$, we can choose a $\left(\bar{D} ; b_{s}, \xi\right)$-optimal strategic function $F^{*}\left(s, N ; \bar{D} ; b_{s}, \xi\right)$ on the basis of Lemma 6.3.1. If we apply the principle of optimality in dynamic programming, in connection with (4.5), on the basis of the result of Theorem 6.2 , we have the relation

$$
\begin{aligned}
U\left(F^{*}\left(s, N ; \bar{D} ; b_{s}, \xi\right) ; b_{s}, \xi\right) \\
\quad=\max _{F(s, N ; \bar{D})} U\left(F(s, N ; \bar{D}) ; b_{s}, \xi\right) \\
\quad=\frac{1}{N-s+1} \cdot \max _{f_{s}\left(e_{s} ; \bar{D}\right)}\left[U\left(f_{s}\left(e_{s} ; \bar{D}\right) ; b_{s}, \xi\right)\right.
\end{aligned}
$$




$$
\begin{aligned}
& \left.+(N-s) \cdot \max _{F(s+1, N ; \bar{D})} \underset{\eta_{n-1}}{E}\left\{U\left(F(s+1, N ; \bar{D}) ; B_{s+1}, \xi\right) \mid b_{s}, f_{s}\left(e_{s} ; \bar{D}\right)\right\}\right] \\
= & \frac{1}{N-s+1} \cdot \max _{f_{s}\left(e_{s} ; \bar{D}\right)}\left[U\left(f_{s}\left(e_{s} ; \bar{D}\right) ; b_{s}, \xi\right)\right. \\
& \left.+(N-n) \cdot E_{\eta_{n+1}}^{E}\left\{U\left(F^{*}(s+1, N ; \bar{D} ; \bar{D}, \xi) ; B_{s+1}, \xi\right) \mid b_{s}, f_{s}\left(e_{s} ; \bar{D}\right)\right\}\right],
\end{aligned}
$$

with respect to each $b_{s}$ in the domain $\bar{D}$.

If we assign any $b_{s}$ in the domain $\bar{D}$, we can choose a function $f_{s}^{*}\left(e_{s} ; \bar{D} ; b_{s}, \xi\right)$ which maximizes the $\left(b_{s}, \xi\right)$-criterion functional $U\left(F(s, N ; \bar{D}) ; b_{s}, \xi\right)$ in (6.16). Hence, we can also make such a function $\hat{f}_{s}\left(e_{s} ; \bar{D}\right)$ as may cause the relation

$$
\hat{f}_{s}\left(b_{s} ; \bar{D}\right)=f_{s}^{*}\left(b_{s} ; \bar{D} ; b_{s}, \xi\right)
$$

to be satisfied with respect to each $b_{s}$ in the domain $\bar{D}$ (see Fig. 6.3).

Let $\hat{F}(s, N ; \bar{D})$ be a strategic function composed of the strategic functions $\hat{f}_{s}\left(e_{s} ; \bar{D}\right)$ and $F^{*}(s+1, N ; \bar{D} ; \bar{D}, \xi)$, i. e. $\left\{\hat{f}_{s}\left(e_{s} ; \bar{D}\right), F^{*}(s+1, N ; \bar{D} ; \bar{D}, \xi)\right\}$. Then, we have

$$
\begin{aligned}
U\left(\hat{F}(s, N ; \bar{D}) ; b_{s}, \xi\right) & =U\left(\left\{\hat{f}_{s}\left(e_{s} ; \bar{D}\right), F^{*}(s+1, N ; \bar{D} ; \bar{D}, \xi)\right\} ; b_{s}, \xi\right) \\
& =U\left(\left\{\hat{f}_{s}\left(b_{s} ; \bar{D}\right), F^{*}(s+1, N ; \bar{D} ; \bar{D}, \xi)\right\} ; b_{s}, \xi\right) \\
& =U\left(\left\{f_{s}^{*}\left(b_{s} ; \bar{D} ; b_{s}, \xi\right), F^{*}(s+1, N ; \bar{D} ; \bar{D}, \xi)\right\} ; b_{s}, \xi\right) \\
& =U\left(\left\{f_{s}^{*}\left(e_{s} ; \bar{D} ; b_{s}, \xi\right), F^{*}(s+1, N ; \bar{D} ; \bar{D}, \xi)\right\} ; b_{s}, \xi\right) \\
& =U\left(F^{*}\left(s, N ; \bar{D} ; b_{s}, \xi\right) ; b_{s}, \xi\right)
\end{aligned}
$$

from (6.17). And we have

$$
\hat{F}(s, N ; \bar{D}) \equiv\left\{\hat{f}_{s}\left(e_{s} ; \bar{D}\right), F^{*}(s+1, N ; \bar{D} ; \bar{D}, \xi)\right\} \in \mathfrak{F}^{*}\left(s, N ; \bar{D} ; b_{s}, \xi\right)
$$

with respect to any $b_{s}$ in the domain $\bar{D}$. That is, we have

$$
\hat{F}(s, N ; \bar{D}) \in \bigcap_{b_{s}=\bar{D}} \mathfrak{F}^{*}\left(s, N ; \bar{D} ; b_{s}, \xi\right)=\mathfrak{F}^{*}(s, N ; \bar{D} ; \bar{D}, \xi) \text {. }
$$

According to the above conclusions (A) and (B), we can admit this theorem is true.

Q.E.D.

Now, we can conduct the stochastic dynamic programming by means of the forward working algorithm on the basis of the following relation:

$$
\begin{aligned}
& \underset{\eta_{n}}{E}\left\{U\left(F^{*}(n, N ; \bar{D} ; \bar{D}, \xi) ; B_{n}, \xi\right)\right\} \\
& =\max _{F(n, N ; \vec{D})} E\left\{U\left(F(n, N ; \bar{D}) ; B_{n}, \xi\right)\right\} \\
& =\frac{1}{N-n+1} \cdot \max _{f_{n}\left(e_{n} ; \bar{D}\right)} \underset{\eta_{n}}{E}\left[U\left(f_{n}\left(e_{n} ; \bar{D}\right) ; B_{n}, \xi\right)\right. \\
& +(N-n) \cdot \max _{F(n+1, N ; \bar{D})} E\left\{\left(U \left(F(n+1, N ; \bar{D}) ; B_{n=1}\right.\right.\right. \\
& =\frac{1}{N-n+1} \cdot \max _{f_{n}\left(e_{n} ; \bar{D}\right)} E\left[U\left(f_{n}\left(e_{n} ; \bar{D}\right) ; B_{n}, \xi\right)\right. \\
& \left.+(N-n) \cdot \underset{n_{n+1}}{E}\left\{U\left(F^{*}(n+1, N ; \bar{D} ; \bar{D}, \xi) ; B_{n+1}, \hat{\xi}\right) \mid B_{n}, f_{n}\left(e_{n} ; \bar{D}\right)\right\}\right] \\
& \left(n=0,1,2, \cdots, N ; B_{0}=b_{0}\right),
\end{aligned}
$$

in place of the relation (4.5). 


\section{§ 7. A Reasonable Utility Function in S. D. M. S.-I}

In order to complete concretely the stochastic dynamic programming on the basis of definitions 4.6 and 4.7 , it is necessary to choose a reasonable utility function $\xi$ in S. D. M.S.-I. For this purpose, let us employ the following three assumptions as the basic attitude in our stochastic dynamic programming. ${ }^{12}$

Assumption 7.1. (i) A utility function $\xi$ is a monotonously increasing and differentiable function on the domain $(-K / r, \infty)$.

(ii) If there is the limitation $\lim _{e \rightarrow-K / r} \xi(e)$, the limitation is equal to $\xi(-K / r)$.

Assumption 7.2. Let $F_{0}(n, N ; \bar{D})$ be a special $\bar{D}$-feasible strategic function so that $F_{0}(n, N ; \bar{D}) \sim 0$, and let us put as $U_{0}\left(N-n+1, b_{n} ; \xi\right)=U\left(F_{0}\left(n, N ; \bar{D} ; b_{n}, \xi\right)\right.$. Then, $U_{0}\left(N-n+1, b_{n} ; \xi\right)$ is a constant, and it is independent of $b_{n}$ larger than $-K / r$ and of non-negative integers $N$ and $n$ so that $n \leqq N$. That is, we can denote as $U_{0}(N-n+1$, $\left.b_{n} ; \xi\right)=U_{0}(\xi)$.

Assumption 7.3. If any common probability density function of the stochastic variables $C_{t}$ 's $(t=0,1,2, \cdots)$, i.e. $\phi$, which satisfies Conditions 3.1-(ii) and 3.1-(iii), is assigned, then, with respect to the probability density function,

(i) the $\left(b_{n}, \xi\right)$-criterion functional $U\left(F(n, N ; \bar{D}) ; b_{n}, \xi\right)$ is finite or negative infinite on $\mathfrak{F}(n, N ; \bar{D})$ in the case of $b_{n} \geqq-K / r$, and

(ii) there is a $(\bar{D} ; \bar{D}, \xi)$-optimal strategic function $F^{*}(n, N ; \bar{D} ; \bar{D}, \xi)$, and the value $U\left(F^{*}(n, N ; \bar{D} ; \bar{D}, \xi) ; b_{n}, \xi\right)$ is a constant, independently of any non-negative integers $n$ and $N$ so that $n \leqq N$ and of any $b_{n}$ larger than $-K / r$.

In Assumptions 7.2 and 7.3, there are not conditions in the case of $b_{n}=-K / r$. However, if $b_{n}=-K / r$, we have $f_{n}\left(b_{n} ; \bar{D}\right)=0$ and also we have $B_{n+1}\left(b_{n}, F(n, N ; \bar{D})\right.$ ) $=b_{n}-K / r$, according to Corollary 5.2 .3 and the relation (3.6). Hence, if $b_{n}=-K / r$, we have

$$
\begin{aligned}
& f_{n}\left(b_{n} ; \bar{D}\right)=f_{n+1}\left(b_{n+1} ; \bar{D}\right)=\cdots=f_{N}\left(b_{N} ; \bar{D}\right)=0 \\
& P\left\{\bigcap_{n+1 \leqq s \leqq N \div 1}\left(B_{s}=b_{n}\right) \mid b_{n}=-K / r, F(n, N ; \bar{D})\right\}=1, \quad U\left(F(n, N ; \bar{D}) ; b_{n}, \xi\right)=0,
\end{aligned}
$$

with respect to any $\bar{D}$-feasible strategic function $F(n, N ; \bar{D})$ and any $\left(b_{n}, \xi\right)$-criterion functional. Therefore, it is not necessary to choose a $(\bar{D} ; \bar{D}, \xi)$-optimal strategic function in case of $b_{n}=-K / r$.

Now, Assumption 7.1 is agreed to be reasonable and necessary in general economic fields.

Assumptions 7.2 and 7.3 may be recognized to be reasonable from a point of view of the 3rd approach in Section 1,2) since these Assumptions reflect the following actual

1) The following assumptions are employed according to a point of view of the 3rd approach in Section 1, on the basis of our intuition. All of these assumptions are not always necessary and there are overlapping parts in these assumptions. More arranged assumptions are shown in the end of this section.

2) Actual manner of making a utility function on the basis of Assumptions 7.2. and 7.3 is different from the manner of Charns, Dreze and Miller [5]. But, the fundamental concept of Assumptions 7.2 and 7.3 coincides with their concept on the third approach stated in Section 1. 
economic situations.

It is commonly agreed that even if we employ a reasonable strategic function through infinite future, (i) the more remote the truncated last time of multi-stage stochastic strategy-making, the larger the probability by which the reasonable strategic function will produce a large profit, and (ii) the greater the initial capital money, also the larger the probability by which the reasonable strategic function will produce a large profit. Hence, it seems reasonable, from the point of view of the 3rd approach in Section 1, to measure the degree of effectiveness of the strategic functions $F_{0}(n, N ; \bar{D})$ and $F^{*}(n, N ; \bar{D} ; \bar{D}, \xi)$ to be independent of $b_{n}$ and $N-n+1$. If $U\left(F_{0}(n, N ; \bar{D}) ; b_{n}, \xi\right)$ or $U\left(F^{*}(n, N ; \bar{D} ; \bar{D}, \xi) ; b_{n}, \xi\right)$ is subject to the length of period of programming $N-n+1$ or the initial condition $b_{n}$, the $\left(b_{n}, \xi\right)$-criterion functional $U\left(F(n, N ; \bar{D}) ; b_{n}, \xi\right)$ on $\mathfrak{F}(n, N ; \bar{D})$ confuses the effectiveness of the activity due to the length of period of programming $N-n+1$ or due to the initial condition $b_{n}$ with the effectiveness of the strategic function $F_{0}(n, N ; \bar{D})$ or $F^{*}(n, N ; \bar{D} ; \bar{D}, \xi)$ itself, respectively.

Now, let us search a utility function which satisfies Assumptions 7.1 through 7.3.

First, we have the following:

THEOREM 7.1. If a utility function $\xi$ satisfies Assumptions 7.1 and $7.2 \mathrm{in}$ S.D.M.S.$I$, the utility function is denoted by

$$
\xi(e)=A \cdot \log (e+K / r)+S(\log (e+K / r))+L \quad(e>-K / r) .
$$

Where, $A$ is a positive constant, and $L$ is any constant, and $S(u)$ is a differentiable periodic function which has a periodic time $\log (1+r)$ and satisfies the following relation:

$$
A+S^{\prime}(\log (e+K / r))>0 \quad(e>-K / r) .
$$

Proof. If we admit Assumption 7.2 and employ a special $\bar{D}$-feasible strategic function $F_{0}(n, N ; \bar{D})$ so that $F_{0}(n, N ; \bar{D}) \sim 0$, we have

$$
\begin{aligned}
& U_{0}(\xi) \\
= & \frac{1}{N-n+1} \cdot\left[E_{n_{N-1}}^{E}\left\{\xi\left(B_{N+1}\right) \mid b_{n}, F_{0}(n, N ; \bar{D})\right\}-\xi\left(b_{n}\right)\right] \\
= & \frac{\xi\left(\left(b_{n}+K / r\right) \cdot \prod_{s=n}^{N}\left(1+r+\left(C_{s}-r\right) \cdot h_{s}\left(B_{s}\left(b_{n}, F_{0}(n, N ; \bar{D}) ; \bar{D}\right)\right)\right)-K / r\right)-\xi\left(b_{n}\right)}{N-n+1} \\
= & \frac{\xi\left(\left(b_{n}+K / r\right) \cdot(1+r)^{N-n+1}-K / r\right)-\xi\left(b_{n}\right)}{N-n+1}, \quad\left(b_{n}>-K / r\right),
\end{aligned}
$$

due to (5.28).

Now, for the sake of simplicity, in some cases, let us employ the following notations.

$$
e+K / r=\tilde{e}, \quad \xi(e)=\tilde{\xi}(e+K / r)=\tilde{\xi}(\tilde{e}), \quad A=U_{0}(\xi) / \log (1+r) .
$$

Then, we have

$$
(N-n+1) \cdot U_{0}(\xi)=\tilde{\xi}\left(\tilde{b}_{n} \cdot(1+r)^{N-n+1}\right)-\tilde{\xi}\left(\tilde{b}_{n}\right) \quad\left(\tilde{b}_{n}>0\right)
$$


from (7.4). Let us express the function $\tilde{\xi}(\tilde{e})$ by the form

$$
\tilde{\xi}(\tilde{e})=A \cdot \log \tilde{e}+\bar{S}(\tilde{e})+L \quad(\tilde{e}>0)
$$

with the constant $A$ (defined in (7.5)) and any constant $L$ and a suitable function $\bar{S}(\tilde{e})$. If we apply the function (7.7) to the relation (7.6) in combination with (7.5), we have the relation

$$
\begin{aligned}
(N- & n+1) \cdot U_{0}(\xi) \\
& =A \cdot \log \left(\tilde{b}_{n} \cdot(1+r)^{N-n+1}\right)+\bar{S}\left(\tilde{b}_{n} \cdot(1+r)^{N-n+1}\right)-A \cdot \log \left(\tilde{b}_{n}\right)-\bar{S}\left(\tilde{b}_{n}\right) \\
& =A \cdot(N-n+1) \cdot \log (1+r)+\bar{S}\left(\tilde{b}_{n} \cdot(1+r)^{N-n+1}\right)-\bar{S}\left(\tilde{b}_{n}\right) \\
& =(N-n+1) \cdot U_{0}(\xi)+\bar{S}\left(\tilde{b}_{n} \cdot(1+r)^{N-n+1}\right)-\bar{S}\left(\tilde{b}_{n}\right), \quad\left(\tilde{b}_{n}>0\right) .
\end{aligned}
$$

Therefore, the function $\bar{S}(\tilde{e})(\tilde{e}>0)$ in (7.7) must satisfy the following relation.

$$
\bar{S}\left(\tilde{e} \cdot(1+r)^{N-n+1}\right)-S(\tilde{e})=0 \quad(\tilde{e}>0) .
$$

Let us employ the following transformation.

$$
\log z=u, \quad \bar{S}(z)=S(\log z)=S(u) .
$$

Then, we have

$$
\begin{aligned}
& S(\log \tilde{e}+(N-n+1) \cdot \log (1+r))-S(\log \tilde{e}) \\
& \quad=S(u+(N-n+1) \cdot \log (1+r))-S(u)=0
\end{aligned}
$$

from (7.9) in combination with (7.10). Therefore, the function $S(u)$ in (7.10) has a periodic time $(N-n+1) \cdot \log (1+r)$. And, since the relation (7.9) must be held with respect to any non-negative integers $n$ and $N$ so that $n \leqq N$ according to Assumption 7.2, the function $S(u)$ must have the minimum periodic time $\log (1+r)$.

Therefore, if a function $S(u)$ is a differentiable periodic function which has a periodic time $\log (1+r)$, the function

$$
\bar{S}(\tilde{e})=S(\log \tilde{e})
$$

is a solution of the functional equation (7.9).

If we substitute the relation (7.12) for (7.7), we have

$$
\xi(e)=\tilde{\xi}(\tilde{e})=A \cdot \log \tilde{e}+S(\log \tilde{e})+L=A \cdot \log (e+K / r)+S(\log (e+K / r))+L
$$

with a differentiable periodic function $S(u)$ which has a periodic time $\log (1+r)$.

Now, we have $\xi^{\prime}(e)>0$ from Assumption 7.1. Therefore, it is necessary to hold the following relation

$$
(e+K / r) \cdot \xi^{\prime}(e)=A+S^{\prime}(\log (e+K / r))>0 \quad(e>-K / r) .
$$

Furthermore, we have $A=U_{0}(\xi) / \log (1+r)>0$, since $U_{0}(\xi)$ is positive according to the definition (7.4) in combination with Assumption 7.1, and $\log (1+r)$ must be positive due to assumption $r>0$ in Condition 3.2-(i).

Q.E.D.

Next, we have the following:

THEOREM 7.2. If (i) a utility function $\xi$ satisfies Assumptions 7.1 through 7.3 in S. D. M.S.-I, and (ii) a number $h^{*}$ produced by the relation 


$$
\max _{0 \leqq h \leqq \alpha} E\{\log (1+r+(C-r) \cdot h)\}=\underset{\psi}{E}\left\{\log \left(1+r+(C-r) \cdot h^{*}\right)\right\}
$$

is positive, ${ }^{1)}$ then the utility function is denoted as

$$
\xi(e)=A \cdot \log (e+K / r)+L \quad(e>-K / r),
$$

where $\alpha=(1+r) /(\beta+r)$, and $A$ is a positive number, ${ }^{2)}$ and $L$ is any constant.

Proof. As the first step, let us verify the following

LEMMA 7.2.1. In S.D.M.S.-I, if we employ a utility function (7.16), then (i) the value $U\left(F^{*}(0, N ; \bar{D} ; \bar{D}, \xi) ; b_{0}, \xi\right)\left(b_{0}>-K / r\right)$ is a constant $P^{*}$ which is independent of $b_{0}$ and $N$, and (ii) $a(\bar{D} ; \bar{D}, \tilde{s})$-optimal strategic function $F^{*}(0, N ; \bar{D} ; \bar{D}, \xi)$ is denoted as

$$
F^{*}(0, N ; \bar{D} ; \bar{D}, \xi) \sim(e+K / r) \cdot h^{*} \quad(e>-K / r)
$$

with a constant $h^{*}$ defined by (7.15).

Proof of Lemma 7.4.1. Let us verify this lemma by means of the mathematical induction.

(a) We can easily admit, due to (5.27), that if the utility function (7.16) is employed, then (i) there is a value $h^{*}$, so that

$$
\begin{aligned}
& U\left(F^{*}(N, N ; \bar{D} ; \bar{D}, \xi) ; b_{N}, \xi\right) \\
& =\max _{0 \leqq h_{N} \leqq \alpha}\left[A \cdot{ }_{\psi_{N}}^{E}\left\{\log \left(\left(b_{N}+K / r\right) \cdot\left(1+r+\left(C_{N}-r\right) \cdot h_{N}\right)\right)\right)\right\} \\
& \left.-A \cdot \log \left(b_{N}+K / r\right)\right] \\
& =\max _{0 \leqq h_{N} \leqq \alpha} A \cdot \underbrace{}_{\psi_{N}}\left\{\log \left(1+r+\left(C_{N}-r\right) \cdot h_{N}\right)\right\} \\
& =A \cdot \underset{\psi_{N}}{E}\left\{\log \left(1+r+\left(C_{N}-r\right) \cdot h^{*}\right)\right\} \\
& =\operatorname{def} . P^{*}
\end{aligned}
$$

holds true uniformly independent of any $b_{N}$ larger than $-K / r$, and (ii) we can denote a $(\bar{D} ; \bar{D}, \xi)$-optimal strategic function as $F^{*}(N, N ; \bar{D} ; \bar{D}, \xi)=\left(e_{N}+K / r\right) \cdot h^{*}$.

(b) Next, let us assume that there is a $(\bar{D} ; \bar{D}, \xi)$-optimal strategic function $F^{*}(s+1, N ; \bar{D} ; \bar{D}, \xi) \sim(e+K / r) \cdot h^{*}$ with a constant $h^{*}$ so that $U\left(F^{*}(s+1, N ; \bar{D} ; \bar{D}, \xi)\right.$; $\left.b_{s+1}, \xi\right)=A \cdot \underset{\boldsymbol{\phi}}{E}\left\{\log \left(1+r+(C-r) \cdot h^{*}\right)\right\}\left(b_{s+1}>-K / r\right)$. Then, by means of the principle of optimality in dynamic programming in which we obtained the relation (6.21), we have the following relation:

1) In this theorem, the assumption $h^{*}>0$ is necessary. That is, in the case of $h^{*}=0$, Assumption 7.3 coincides with Assumption 7.2, and we have only a conclusion due to Theorem 7.1. Therefore, it is necessary that there is a case of $h^{*}>0$ in S. D. M.S.-I, in order that we have a utility function (7.16). The condition $h^{*}>0$ does not constrain our general argument. Assumption 7.3 is employed with respect to any common probability density function of the stochastic variables $C_{t}$ 's, i.e. $\psi$, which satisfies Conditions 3.1 -(ii) and 3.1-(iii). We can easily admit that there is a positive $h^{*}$ defined in (7.15) in connection with a probability density function $\phi$ which satisfies Conditions $3.1-$ (ii) and 3.1-(iii).

2) A is defined by $A=U_{0}(\xi) / \log (1+r)$ in (7.5). However, $A$ may be any positive number, since $U_{0}(\xi)$ may be any assigned positive number. 


$$
\begin{aligned}
U\left(F^{*}\right. & \left.(s, N ; \bar{D} ; \bar{D}, \xi) ; b_{s}, \xi\right) \\
= & \max _{0 \leqq h_{s} \cong \alpha}-\frac{A}{N-s+1} \cdot\left[\underset{\psi_{s}}{E}\left\{\log \left(\left(b_{s}+K / r\right) \cdot\left(1+r+\left(C_{s}-r\right) \cdot h_{s}\right)\right)\right)\right\} \\
& -\log \left(b_{s}+K / r\right)+(N-s) \cdot \underset{\psi}{E}\left\{\log \left(1+r+(C-r) \cdot h^{*}\right)\right\} \\
= & A \cdot \underset{\psi}{E}\left\{\log \left(1+r+(C-r) \cdot h^{*}\right)\right\}=P^{*} .
\end{aligned}
$$

By means of the mathematical induction, we have a $(\bar{D} ; \bar{D}, \xi)$-optimal strategic function $F^{*}(s, N ; \bar{D} ; \bar{D}, \xi) \sim(e+K / r) \cdot h^{*}$. Hence, we can admit this lemma.

Next, we have the following

LEMMA 7.2.1. Q.E. D.

LEMмA 7.2.2. In S.D.M.S.-I, if a utility function $\xi$ satisfies Assumptions 7.1 through 7.3 , we have

$$
U\left(F^{*}(0, N ; \vec{D} ; \bar{D}, \xi) ; b_{0}, \xi\right)=A \cdot \underset{\psi}{E}\left\{\log \left(1+r+(C-r) \cdot h^{*}\right)\right\}=P^{*}
$$

with respect to any $b_{0}(>-K / r)$ and any non-negative integer $N$, with constants $h^{*}$ and $P^{*}$ defined in (7.18).

ProOF of LEMma 7.2.2. Since the utility function $\xi$ satisfies Assumptions 7.1 and 7.2, the utility function $\xi$ is denoted by (7.2) due to Theorem 7.1. If we substitute (5.28) for (4.4), and also substitute the utility function (7.2) for the substituted relation (4.4), then, we have

$$
\begin{aligned}
& U\left(F^{*}(0, N ; \bar{D} ; \bar{D}, \xi) ; b_{0}, \xi\right) \\
& =\frac{1}{N+1} \cdot \max _{0 \leq H(0, N) \leq \alpha}\left[\underset { \psi _ { 0 } } { E } \left\{\cdots \left\{\underset { \psi _ { N } } { E } \left\{\xi \left(\left(b_{0}+K / r\right)\right.\right.\right.\right.\right. \\
& \left.\left.\left.\left.\left.\cdot \prod_{t=0}^{N}\left(1+r+\left(C_{t}-r\right) \cdot h_{t}\left(B_{t}\left(b_{0}, H(0, N)\right)\right)-K / r\right)\right)\right\}\right\} \cdots\right\}-\xi\left(b_{0}\right)\right] \\
& =\frac{1}{N+1} \cdot \max _{0 \leq H(0, N) \leq \alpha}\left[A \cdot \underset { \psi _ { 0 } } { E } \left\{\cdots \left\{\underset { \psi _ { \mathbf { N } } } { E } \left\{\sum _ { i = 0 } ^ { N } \operatorname { l o g } \left(1+r+\left(C_{t}-r\right)\right.\right.\right.\right.\right. \\
& \left.\left.\left.\left.\cdot h_{t}\left(B_{t}\left(b_{0}, H(0, N)\right)\right)\right)\right\}\right\} \cdots\right\}+\underset{\psi_{0}}{E}\left\{\cdots \left\{\underset { \psi _ { N } } { E } \left\{S\left(\log \left(b_{0}+K / r\right)\right)\right.\right.\right. \\
& \left.\left.\left.\left.\left.+\sum_{t=0}^{N} \log \left(1+r+\left(C_{t}-r\right) \cdot h_{t}\left(B_{t}\left(b_{0}, H(0, N)\right)\right)\right)\right)-S\left(\log \left(b_{0}+K / r\right)\right)\right\}\right\} \cdots\right\}\right] \\
& =\text { def. } P^{*}, \quad\left(B_{0}\left(b_{0}, H(0, N)\right)=b_{0}\right),
\end{aligned}
$$

independently of $b_{0}(>-K / r)$ and of non-negative integer $N$, due to Assumption 7.3.

Now, since the function $S(u)$ is a differentiable periodic function which has a periodic time $\log (1+r)$ according to Theorem 7.1, there is a finite number $\rho$ so that $-\rho<S(u)<\rho$ holds true on the domain $(-\infty, \infty)$. That is, we have

$$
\begin{aligned}
-\rho<\underset{\psi_{0}}{E}\{\cdots & \left\{\underset { \psi _ { N } } { E } \left\{S\left(\log \left(b_{0}+K / r\right)\right)+\sum_{t=0}^{N} \log \left(1+r+\left(C_{t}-r\right)\right.\right.\right. \\
& \left.\left.\left.\left.\cdot h_{t}\left(B_{t}\left(b_{0}, H(0, N)\right)\right)\right)-S\left(\log \left(b_{0}+K / r\right)\right)\right\}\right\} \cdots\right\}<\rho
\end{aligned}
$$


with respect to any $b_{0}(>-K / r)$, any non-negative integer $N$ and any function $H(0, N)$ so that $0 \lesssim H(0, N) \lesssim \alpha$. Therefore, if we notice Assumption 7.3 and the conclusion of Lemma 7.2 .1 in connection with the relations (7.21) and (7.22), we can easily admit the relation

$$
\begin{gathered}
\lim _{N \rightarrow \infty} U\left(F^{*}(0, N ; \bar{D} ; \bar{D}, \xi) ; b_{0}, \xi\right) \\
=\lim _{N \rightarrow \infty} \max _{0 \leq H(0, N) \leq \alpha} \frac{A}{N+1} \cdot{ }_{\psi_{0}}^{E}\left\{\cdots \left\{\sum _ { \psi _ { N } } ^ { E } \left\{\sum _ { t = 0 } ^ { N } \operatorname { l o g } \left(1+r+\left(C_{t}-r\right)\right.\right.\right.\right. \\
\left.\left.\left.\left.\cdot h_{t}\left(B_{t}\left(b_{0}, H(0, N)\right)\right)\right)\right\}\right\} \cdots\right\} \\
=A \cdot \underset{\psi}{E}\left\{\log \left(1+r+(C-r) \cdot h^{*}\right)\right\}=P^{*} .
\end{gathered}
$$

Since $U\left(F^{*}(0, N ; \bar{D} ; \bar{D}, \xi) ; b_{0}, \xi\right)$ is uniformly independent of $b_{0}$ larger than $-K / r$ and of non-negative integer $N$ according to Assumption 7.3, we can admit this lemma is true from (7.23).

LEMMA 7.2.2. Q. E. D.

Furthermore, we have the following

LEMMA 7.2.3. If (i) a function $S(u)(-\infty<u<\infty)$ is a differentiable periodic function which is not any constant, and (ii) the function $S(u)$ has a periodic time $\log (1+r)$, and (iii) a number $h^{*}$ produced by the relation (7.15) is positive, then there is a number $b_{0}$ larger than $-K / r$ so that

$$
\begin{aligned}
\max _{0 \leqq h \leqq \alpha}[ & E\left\{\operatorname{Elog}\left(1+r+\left(C_{0}-r\right) \cdot h\right)\right\} \\
& \quad+\underset{\psi_{0}}{E}\left\{S\left(\log \left(b_{0}+K / r\right)+\log \left(1+r+\left(C_{0}-r\right) \cdot h\right)\right)\right\} \\
& \left.\quad-S\left(\log \left(b_{0}+K / r\right)\right)\right] \\
< & \underset{\psi_{0}}{E}\left\{\log \left(1+r+\left(C_{0}-r\right) \cdot h^{*}\right)\right\}
\end{aligned}
$$

holds true.

ProOF OF LEMMA 7.2.3. According to the assumptions (i) and (ii) in this lemma, we can choose a positive number $\delta$ and a number $u^{*}$ so that (i) $S(u) \leqq S\left(u^{*}\right)$ may be held on the domain $(-\infty, \infty)$, and (ii) $S\left(u^{*}+\Delta u\right)<S\left(u^{*}\right)$ may be held on any $\Delta u$ such that $0<\Delta u<\delta$, (see Fig. 7.1).

Let us choose a number $b_{0}$ such that $\log \left(b_{0}+K / r\right)=u^{*}$. Since we have $S\left(\log \left(b_{0}\right.\right.$ $+K / r)=S\left(\log \left(b_{0}+K / r\right)+\log (1+r)\right)$ due to the assumption (i) in this lemma, and we have also $\phi(e)>0$ on the domain $(-\beta, \gamma)$ due to Condition 3.1-(iii), we have the relation

$$
\begin{aligned}
& \underset{\psi_{0}}{E}\left\{S\left(\log \left(b_{0}+K / r\right)+\log \left(1+r+\left(C_{0}-r\right) \cdot h\right)\right)\right\} \\
& \quad=\int_{-\beta}^{r} S\left(\log \left(b_{0}+K / r\right)+\log (1+r+(e-r) \cdot h)\right) \cdot \psi_{0}(e) \cdot d e \\
& \quad<S\left(\log \left(b_{0}+K / r\right)+\log (1+r)\right)=S\left(\log \left(b_{0}+K / r\right)\right)
\end{aligned}
$$

on any positive number $h$ smaller than $\alpha$. (7.25) shows this lemma is true.

LEMMA 7.2.3. Q. E. D.

Lemma 7.2.3 shows that if (i) we employ a utility function (7.2) in which the 


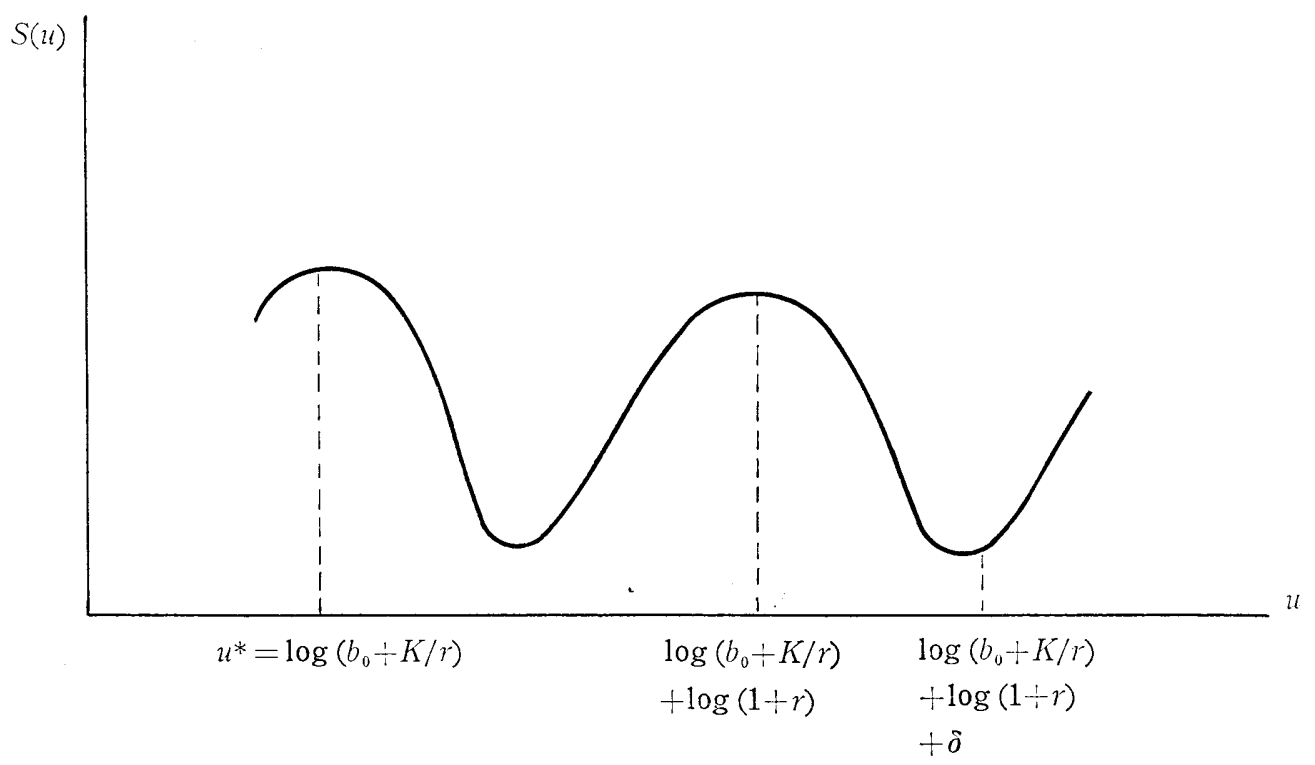

Fig. 7.1.

function $S(u)$ is a periodic function which has the periodic time $\log (1+r)$ but is not any constant, and (ii) such number $h^{*}$ as (7.15) holds true is positive, then there is a number $b_{0}$ (in the domain $(-K / r, \infty)$ ) so that the relation

$$
U\left(F^{*}(0,0 ; \bar{D} ; \bar{D}, \xi) ; b_{0}, \xi\right)=A \cdot \underset{\psi}{E}\left\{\log \left(1+r+(C-r) \cdot h^{*}\right)\right\}
$$

cannot hold true. Noting Lemma 7.2.2, we can easily admit that if $S(u)$ is not a constant, Assumption 7.3 cannot be employed.

THEOREM 7.2. Q. E. D.

Now, strictly speaking, all Assumptions 7.1 through 7.3 are not always necessary, and there are overlapping parts in these assumptions. That is,

(I) We can easily admit from Theorem 7.1 that if Assumptions 7.1-(i) and 7.2 are true in S. D. M. S.-I, Assumption 7.1-(ii) is not necessary, since we have

$$
\lim _{e \rightarrow-K / r} \xi(e)=\lim _{e \rightarrow-K / r}\{A \cdot \log (e+K / r)+S(\log (e+K / r))+L\}=-\infty \quad(A>0) .
$$

(II) Next, we have the following

THEOREM 7.3. Let $F_{0}(n, N ; \bar{D})$ be a special $\bar{D}$-feasible strategic function so that $F(n, N ; \bar{D}) \sim 0$, and let $U_{0}\left(N-n+1, b_{n} ; \xi\right)$ be $U\left(F_{0}(n, N ; \bar{D}) ; b_{n}, \xi\right)$. If the function $U_{0}\left(N-n+1, e_{n} ; \xi\right)$ is independent of $e_{n}$, then the function $U_{0}\left(N-n+1 ; e_{n} ; \xi\right)$ is also independent of $N-n+1(N=0,1,2, \cdots ; n=0,1,2, \cdots, N)$.

Proof. Let us put as $\left(b_{n}+K / r\right) \cdot(1+r)^{N-n+1}-K / r=b_{N}\left(b_{n}\right)$ and put as $U_{0}(N-n+1$, $\left.e_{n} ; \xi\right)=U_{0}(N-n+1 ; \xi)$ on the basis of the assumption of this theorem. Then, we have

$$
(N-n+1) \cdot U_{0}(N-n+1 ; \xi)=\xi\left(b_{N+1}\left(b_{n}\right)\right)-\xi\left(b_{n}\right) \quad\left(b_{n}>-K / r\right),
$$

applying the relation (7.4). If we put as $N=1$ and $n=0$ in (7.28), we have

$$
\begin{aligned}
2 \cdot U_{0}(2 ; \xi) & =\xi\left(b_{2}\left(b_{0}\right)\right)-\xi\left(b_{1}\left(b_{0}\right)\right)+\xi\left(b_{1}\left(b_{0}\right)\right)-\xi\left(b_{0}\right) \\
& =U_{0}(1 ; \xi)+U_{0}(1 ; \xi) \\
& =2 \cdot U_{0}(1 ; \xi),
\end{aligned}
$$


applying the relation (7.28) again. And also we have

$$
U_{0}(2 ; \xi)=U_{0}(1 ; \xi) \text {. }
$$

Next, if $U_{0}(n ; \xi)=U_{0}(1 ; \xi)$ holds true, then we have

$$
\begin{aligned}
(n+1) \cdot U_{0}(n+1 ; \xi) & =\xi\left(b_{n+1}\left(b_{0}\right)\right)-\xi\left(b_{1}\left(b_{0}\right)\right)+\xi\left(b_{1}\left(b_{0}\right)\right)-\xi\left(b_{0}\right) \\
& =n \cdot U_{0}(n ; \xi)+U_{0}(1 ; \xi) \\
& =(n+1) \cdot U_{0}(1 ; \xi)
\end{aligned}
$$

applying the relation (7.28), and we have also

$$
U_{0}(n+1 ; \xi)=U_{0}(1 ; \xi) \quad(n=0,1,2, \cdots) .
$$

Applying the mathematical induction, we can admit this theorem.

(III) Furthermore, we have the following

THEOREM 7.4. If (i) a utility function $\xi(e)$ satisfies Assumptions 7.1-(i) and 7.2, and (ii) $b_{n}$ is larger than $-K / r$, then (i) a $\left(b_{n}, \xi\right)$-criterion functional $U(F(n, N ; \bar{D})$; $\left.b_{n}, \xi\right)$ can be defined on $\mathfrak{F}(n, N ; \bar{D})$, and (ii) it is upper bounded on the set $\mathfrak{F}(n, N ; \bar{D})$, uniformly with respect to any $b_{n}$ larger than $-K / r$ and with respect to any nonnegative integers $n$ and $N$ so that $n \leqq N$.

Proof. According to Corollary 5.3.3, in combination with the notation (5.24), we have

$$
\begin{array}{r}
P\left\{\bigcap_{n \div 1 \leqq t \leqq N-1}\left(B_{t}>-K / r\right) \mid b_{n}>-K / r, 0 \lesssim H(0, N) \lesssim \alpha\right\}=1 \\
(1+r) /(\beta+r)=\alpha .
\end{array}
$$

And, by means of the same manner as we have (5.28), we have

$$
\begin{gathered}
B_{N+1}\left(b_{n}, F(n, N ; \bar{D})\right)+K / r \\
=B_{N+1}\left(b_{n}>-K / r, 0 \lesssim H(n, N) \lesssim \alpha\right)+K / r \\
=\left(b_{n}+K / r\right) \cdot\left(1+r+\left(C_{n}-r\right) \cdot h_{n}\left(b_{n}\right)\right) \cdot\left(1+r+\left(C_{n+1}-r\right) \cdot h_{n+1}\left(B_{n+1}\right)\right) \\
\quad \cdots \cdot\left(1+r+\left(C_{N}-r\right) \cdot h_{N}\left(B_{N}\right)\right) .
\end{gathered}
$$

Since Assumption 7.2 is true, the utility function $\xi(e)$ is denoted by (7.2) due to Theorem 7.1. Hence, we have

$$
\begin{aligned}
U\left(F(n, N ; \bar{D}) ; b_{n}, \xi\right) & \\
= & \frac{A}{N-\frac{A}{n+1}} \cdot \underset{\psi_{n}}{E}\left\{\cdots \left\{\underset { \psi _ { N } } { E } \left\{\sum _ { t = n } ^ { N } \operatorname { l o g } \left(1+r+\left(C_{t}-r\right)\right.\right.\right.\right. \\
& \left.\left.\left.\left.\left.\cdot h_{t}\left(B_{t}\left(b_{n}, H(n, N ; \bar{D})\right)\right)\right)\right\}\right\}\right\}\right\} \\
& +\frac{1}{N-n+1} \cdot \underset{\psi_{n}}{E}\left\{\cdots \left\{\psi _ { \psi _ { N } } \left\{S\left(\log \left(b_{n}+K / r\right)\right)\right.\right.\right. \\
& +\sum_{t=n}^{N} \log \left(1+r+\left(C_{t}-r\right) \cdot h_{t}\left(B_{t}\left(b_{n}, H(n, N ; \bar{D})\right)\right)\right) \\
& \left.\left.\left.-S\left(\log \left(b_{n}+K / r\right)\right)\right\}\right\} \cdots\right\} \quad\left(B_{n}\left(b_{n}, H(n, N ; \bar{D})\right)=b_{n}\right) .
\end{aligned}
$$


Since, $S(u)$ is a periodic function on $(-\infty, \infty)$ which has a periodic time $\log (1+r)$, we have

$$
\begin{aligned}
U\left(F(n, N ; \bar{D}) ; b_{n}, \xi\right) \leqq & A \cdot \log (1+r+(\gamma-r) \cdot \alpha) \\
& +\frac{1}{N-n+1} \cdot\left\{\max _{u} S(u)-\min _{u} S(u)\right\} .
\end{aligned}
$$

And, we can admit that $U\left(F(n, N ; \bar{D}) ; b_{n}, \xi\right)$ is upper bounded on $\mathfrak{F}(n, N ; \bar{D})$ if $b_{n}$ $>-K / r$, with respect to any non-negative integers $n$ and $N$ so that $n \leqq N$. Q. E. D.

According to the previous considerations (I) through (III), we can admit that, for the purpose of choosing a reasonable utility function from the point of view of the 3rd approach in Section 1 in S. D. M. S.-I, Assumptions 7.1 through 7.3 may be substituted by the following three assumptions.

Assumption 7.4. A utility function $\xi$ is a monotonously increasing and differentiable function on the domain $(-K / r, \infty)$.

ASSUMPTION 7.5. Let $F_{0}(n, N ; \bar{D})$ be a special $\bar{D}$-feasible strategic function so that $F_{0}(n, N ; \bar{D}) \sim 0$, and let $U_{0}\left(N-n+1, b_{n} ; \xi\right)$ be $U\left(F_{0}(n, N ; \bar{D}) ; b_{n}, \xi\right)$. Then, $U_{0}\left(N-n+1, b_{n}, \xi\right)$ is independent of $b_{n}$ in the domain $(-K / r, \infty)$.

Assumption 7.6. There is a $(\bar{D} ; \bar{D}, \xi)$-optimal strategic function $F^{*}(n, N ; \bar{D} ; \bar{D}, \xi)$, and $U\left(F^{*}(n, N ; \bar{D} ; \bar{D}, \xi) ; b_{n}, \xi\right)$ is a constant, independently of $b_{n}$ in the domain $(-K / r$, $\infty)$ and of any non-negative integers $n$ and $N$ so that $n \leqq N$.

\section{§8. Some Comments on the Utility Function $\xi(e)=A \cdot \log (e+K / r)+L$}

(A) First, let us see that the utility function $\xi(e)=A \cdot \log (e+K / r)+L$ is really applicable in the problem of multi-stage stochastic strategy-making. That is, let us see the utility function has important significance not only from the point of view of the 3rd approach in Section 1 but from the point of view of the 2nd approach in the section.

A value of criterion functional (4.4) is only a "expectation" of utility of amount of money which will be obtained at the time $N+1$ through a strategic function $F\left(n, N ; b_{n}\right)$. However, the real goal of the stochastic dynamic programming is not to obtain the expectation of utility of amount of money, since the expectation is only a mathematical form and it cannot always be obtained as a realization at the time $N+1$. If the expectation, at least, does not have a high degree of reliability, the strategy which produces the maximal expectation is inapplicable. It is necessary to notice how reliable is the expectation produced by a stochastic strategy.

Now, let us measure the degree of reliability in obtaining the expectation of a stochastic variable $\frac{1}{N+1} \cdot\left[\xi\left(B_{N+1}\left(b_{0}, F(0, N ; \bar{D})\right)\right)-\xi\left(b_{0}\right)\right]$, i. e. $U\left(F(0, N ; \bar{D}) ; b_{0}, \xi\right)$, by the "coefficient of variation" of the stochastic variable. Then, it seems reasonable that a utility function employed in a stochastic dynamic programming satisfies the following :

CONDITION 8.1. Let $V\{B\}$ be the variance of a stochastic variable B. Then, a utility function $\xi$ satisfies the following relation.

$$
\lim _{N \rightarrow \infty} \frac{\sqrt{V\left\{\frac{1}{N+1} \cdot\left(\xi\left(B_{N+1}\right)-\xi\left(b_{0}\right)\right) \mid b_{0}, F^{*}(0, N ; \bar{D} ; \bar{D}, \xi)\right\}}}{U\left(F^{*}(0, N ; \bar{D} ; \bar{D}, \xi) ; b_{0}, \xi\right)}=0 .
$$


We have the following

THEOREM 8.1. If a utility function $\xi(e)=A \cdot \log (e+K / r)+L$ is employed for the purpose of the stochastic dynamic programming in S.D.M.S.-I, the utility function satisfies Condition 8.1 .

PROOF. In the process of proving Lemma 7.2.1, we have verified that if we employ a utility function $\xi(e)=A \cdot \log (e+K / r)+L$, then we have

$$
F^{*}(0, N ; \bar{D} ; \bar{D}, \xi) \sim(e+K / r) \cdot h^{*}
$$

and

$$
U\left(F^{*}(0, N ; \bar{D} ; \bar{D}, \xi) ; b_{0}, \xi\right)=A \cdot \underset{\psi}{E}\left\{\log \left(1+r+(C-r) \cdot h^{*}\right)\right\} .
$$

Therefore, if the utility function $\xi(e)=A \cdot \log (e+K / r)+L$ is employed, we have also

$$
\begin{gathered}
\log \left(B_{N+1}\left(b_{0}, F^{*}(0, N ; \bar{D} ; \bar{D}, \xi)\right)+K / r\right)-\log \left(b_{0}+K / r\right) \\
=\sum_{t=0}^{N} \log \left(1+r+\left(C_{t}-r\right) \cdot h^{*}\right),
\end{gathered}
$$

according to (5.28) in combination with (8.2).

Now, let $V_{N+1}$ be the variance of the stochastic variable $\frac{A}{N+1} \cdot\left\{\log \left(B_{N+1}\left(b_{0}\right.\right.\right.$, $\left.\left.F^{*}(0, N ; \bar{D} ; \bar{D}, \xi)+K / r\right)-\log \left(b_{0}+K / r\right)\right\}$, and let $v$ be the variance of each stochastic variable $A \cdot \log \left(1+r+\left(C_{t}-r\right) \cdot h^{*}\right)(t=0,1,2, \cdots)$. Then, we have

$$
V_{N+1}=\frac{v}{N+1}
$$

due to (8.4), since the stochastic variables $\log \left(1+r+\left(C_{t}-r\right) \cdot h^{*}\right)$ 's $(t=0,1,2, \ldots)$ are independent with each other according to Condition 3.1-(i). Therefore, the coefficient of variation of the stochastic variable $\frac{A}{N+1} \cdot\left\{\log \left(B_{N+1}\left(b_{0}, F^{*}(0, N ; \bar{D} ; \bar{D}, \xi)+K / r\right)\right.\right.$ $\left.-\log \left(b_{0}+K / r\right)\right\}$ becomes equal to

$$
\frac{\sqrt{v}}{\sqrt{(N+1)} \cdot E_{\psi}\left\{\log \left(1+r+(C-r) \cdot h^{*}\right)\right\}} \cdot
$$

That is, we can admit this theorem.

Q. E. D.

(B) Furthermore, it is interesting to notice the following fact based upon the utility function $A \cdot \log (e+K / r)+L$.

In S. D. M. S.-I, such a profit as may be produced through an activity by means of $x$ is a stochastic realization. On the other hand, such a profit as may be produced through an activity by means of $y$ is not. According to our intuition, it may seem reasonable that the ratio of optimal level of $x_{0}$ to the amount of total capital money $b$, i. e. $x_{0}^{*}(b) / b$, does not decrease according to increase in capital money $b$ [9], since it seems that the larger the total capital money is, the smaller the risk with which a management activity becomes disadvantageous. However, if we make use of the utility function $\xi(e)=A \cdot \log (e+K / r)+L$, the value $x_{0}^{*}(b) / b=f_{0}^{*}(b ; \bar{D} ; \bar{D}, \xi) / b$ does not always decrease. That is, it is easy to see that we have 


$$
\frac{x_{0}^{*}(b)}{b}=\frac{f_{0}^{*}(b ; \bar{D} ; \bar{D}, \xi)}{b}=\frac{(b+K / r) \cdot h^{*}}{b}=\left(1+\frac{K}{r \cdot b}\right) \cdot h^{*} \quad(b>-K / r) .
$$

This relation shows that if $K<0$, our ordinary intuition agrees with the conclusion from the objective stochastic strategy-making by means of the stochastic dynamic programming on the basis of the utility function $\xi(e)=A \cdot \log (e+K / r)+L$. However, if $K>0$, the relation (8.7) shows that our ordinary intuition does not agree with the conclusion.

(C) According to Theorem 7.2 and Lemma 7.2.1, if Assumptions 7.1 through 7.3 are admitted in S.D.M.S.-I, an optimal stochastic strategy at each time $t$ is denoted by $x *\left(b_{t}\right)=\left(b_{t}+K / r\right) \cdot h^{*}$ and $y^{*}\left(b_{t}\right)=b_{t}-x *\left(b_{t}\right)$ in combination with the value $h^{*}$ maximizes $\underset{\psi_{t}}{E}\left\{\log \left(1+r+\left(C_{t}-r\right) \cdot h\right)\right\}$.

If the domain $D(r+(C-r) \cdot h)$ is so small that we can neglect all terms beyond the second degree in the expansion of the function $\log (1+r(c-r) \cdot h)$, with respect to any $h$ in a domain $\left[h^{*}-\delta, h^{*}+\delta\right]$, then we may denote the function approximately as follows :

$$
\log (1+r+(c-r) \cdot h)=r+(c-r) \cdot h-\frac{1}{2} \cdot(r+(c-r) \cdot h)^{2} .
$$

That is, denoting the variance of the stochastic variable $C$ by $\sigma^{2}$, we have also

$$
\underset{\psi}{E}\left\{\log (1+r+(C-r) \cdot h\}=r+(m-r) \cdot h-\frac{1}{2} \cdot\left\{(r+(m-r) \cdot h)^{2}+\sigma^{2} \cdot h^{2}\right\},\right.
$$

for the purpose of making an approximate stochastic strategy.

Hence, an approximate optimal value $h^{*}$ which maximizes (8.9) becomes equal to

$$
h^{*}=\frac{(m-r) \cdot(1-r)}{(m-r)^{2}+\sigma^{2}} .
$$

For example, if we put as $m=0.06, \sigma^{2}=0.03$ and $r=0.04$ in S. D. M.S. - I, the optimal value $h^{*}$ becomes equal to

$$
h^{*}=\frac{0.02 \times 0.96}{(0.02)^{2}+0.03}=0.6316
$$

and the optimal strategy is

$$
\begin{aligned}
f^{*}\left(b_{t} ; \bar{D} ; \bar{D}, \xi\right) & =\max \left[0,0.6316 b_{t}+\frac{0.6316}{0.04} \cdot K\right] \\
& =\max \left[0,0.6316 b_{t}+15.7895 K\right] .
\end{aligned}
$$

\section{§. On the Linear Utility Function and the Bernoulli's Utility Function}

(A) It is generally agreed that the strategy which maximizes expectation of capital money under a stochastic scheme will not always lead a reasonable stochastic strategy. Let us verify this situation objectively from a point of view of the stochatic dynamic programming.

In S.D.M.S.-I, if a monotonously increasing linear utility function $\xi(e)=A$ $\cdot(e+K / r)+L(A>0)$ is employed, a relation 


$$
\begin{aligned}
U\left(F^{*}\right. & \left.(N, N ; \bar{D} ; \bar{D}, \xi) ; b_{N}, \xi\right) \\
& =\max _{0 \leqq h_{N} \leqq \alpha} A \cdot \underset{\psi_{N}}{E}\left\{\left(b_{N}+K / r\right) \cdot\left(1+r+\left(C_{N}-r\right) \cdot h_{N}\right)-\left(b_{N}+K / r\right)\right\} \\
& =\max _{0 \leqq h_{N} \leqq \alpha} A \cdot\left(b_{N}+K / r\right) \cdot\left(\left(1+r+(m-r) \cdot h_{N}\right)-1\right) \\
& =A \cdot\left(b_{N}+K / r\right) \cdot((1+r+(m-r) \cdot \alpha)-1) \\
& =A \cdot\left(b_{N}+K / r\right) \cdot(R-1)
\end{aligned}
$$

is obtained with respect to any assigned $b_{N}$ in the domain $\bar{D}$, putting as $\alpha=(1+r)$ $/(\beta+r)$ and $R=1+r+(m-r) \cdot \alpha$.

Now, let us apply the linear utility function and the relation (9.1) to the relation (6.21) in the case of $n=N-1$. Then, we have

$$
\begin{aligned}
& U\left(F^{*}(N-1, N ; \bar{D} ; \bar{D}, \xi) ; b_{N-1}, \xi\right) \\
& =\max _{0 \leqq h_{N-1} \leqq \alpha} \frac{1}{2} \cdot\left[\underset{\eta_{N}}{E}\left\{\xi\left(B_{N}\right) \mid b_{N-1}, h_{N-1}\right\}-\xi\left(b_{N-1}\right)\right. \\
& \left.+\underset{\eta_{N}}{E}\left\{U\left(F^{*}(N, N ; \bar{D} ; \bar{D}, \xi) ; B_{N}\left(b_{N-1}, h_{N-1}\right), \xi\right)\right\}\right] \\
& =\max _{0 \leqq h_{N-1} \leqq \alpha} \frac{A}{2} \cdot\left[\underset{\psi, V-1}{E}\left\{\left(b_{N-1}+K / r\right) \cdot\left(1+r+\left(C_{N-1}-r\right) \cdot h_{N-1}\right)\right\}-\left(b_{N-1}+K / r\right)\right. \\
& \left.+\underset{\psi_{N-1}}{E}\left\{\left(B_{N-1}+K / r\right) \cdot\left(1+r+\left(C_{N-1}-r\right) \cdot h_{N-1}\right) \cdot(R-1)\right\}\right] \\
& =\max _{0 \leqq h_{N-1} \leqq \alpha} \frac{A}{2} \cdot\left(b_{N-1}+K / r\right) \cdot\left\{\left(\left(1+r+(m-r) \cdot h_{N-1}\right)-1\right)\right. \\
& \left.+\left(1+r+(m-r) \cdot h_{N-1}\right) \cdot(R-1)\right\} \\
& =\begin{array}{c}
A \\
2
\end{array} \cdot\left(b_{N-1}+K / r\right) \cdot\{((1+r+(m-r) \cdot \alpha)-1)+(1+r+(m-r) \cdot \alpha) \cdot(R-1)\} \\
& =\frac{A}{2} \cdot\left(b_{N-1}+K / r\right) \cdot\{(R-1)+R \cdot(R-1)\} \\
& =\frac{A}{2} \cdot\left(b_{N-1}+K / r\right) \cdot(R+1) \cdot(R-1) \\
& =-\frac{A}{2} \cdot\left(b_{N-1}+K / r\right) \cdot\left(R^{2}-1\right)
\end{aligned}
$$

with respect to any assigned $b_{N-1}$ in the domain $\bar{D}$. And similarly, we have also

$$
\begin{aligned}
U\left(F^{*}\right. & \left.(N-2, N ; \bar{D} ; \bar{D}, \xi) ; b_{N-2}, \xi\right) \\
& =\frac{A}{3} \cdot\left(b_{N-2}+K / r\right) \cdot\{(R-1)+R \cdot(R+1) \cdot(R-1)\} \\
& =\frac{A}{3} \cdot\left(b_{N-2}+K / r\right) \cdot\left(R^{2}+R+1\right) \cdot(R-1) \\
& =\frac{A}{3} \cdot\left(b_{N-2}+K / r\right) \cdot\left(R^{3}-1\right)
\end{aligned}
$$

with respect to any assigned $b_{N-2}$ in the domain $\bar{D}$. Furthermore, we have also 


$$
\begin{aligned}
U(F & \left.*(0, N ; \bar{D} ; \bar{D}, \xi) ; b_{0}, \xi\right) \\
& =\frac{A}{N+1} \cdot\left(b_{0}+K / r\right) \cdot\left(R^{N}+R^{N-1}+\cdots+1\right) \cdot(R-1) \\
& =\frac{A}{N+1} \cdot\left(b_{0}+K / r\right) \cdot\left(R^{N+1}-1\right) .
\end{aligned}
$$

Hence, if a linear utility function $\xi(e)=A \cdot(e+K / r)+L$ is employed, we can obtain a $(\bar{D} ; \bar{D}, \xi)$-optimal strategic function

$$
F *(0, N ; \bar{D} ; \bar{D}, \xi) \sim(e+K / r) \cdot \alpha
$$

and we have the relation

$$
\xi\left(B_{N+1}\left(b_{0}, F *(0, N ; \bar{D} ; \bar{D}, \xi)\right)\right)=A \cdot\left(b_{0}+K / r\right) \cdot \prod_{t=0}^{N}\left(1+r+\left(C_{t}-r\right) \cdot \alpha\right)+L,
$$

applying (9.5) to (5.28).

Now, let $\sigma^{2}$ be the variance of the stochastic variables $C_{n}$ 's and let $V_{N+1}$ be the variance of the stochastic variable $\frac{A}{N+1} \cdot\left\{\xi\left(B_{N+1}\left(b_{0}, F^{*}(0, N ; \bar{D} ; \bar{D}, \xi)\right)\right)-\xi\left(b_{0}\right)\right\}$. Then, we have

$$
\begin{aligned}
V_{N+1}=\left\{\frac{A \cdot\left(b_{0}+K / r\right)}{N+1} \cdot(1+r+(m-r) \cdot \alpha)^{N+1}\right\}^{2} \\
\cdot\left\{\left(1+\frac{\sigma^{2} \cdot \alpha^{2}}{(1+r+(m-r) \cdot \alpha)^{2}}\right)^{N-1}-1\right\}
\end{aligned}
$$

from (9.6), since stochastic variables $\left(1+r+\left(C_{t}-r\right) \cdot \alpha\right)$ 's $(t=0,1,2, \cdots, N)$ are independent of each other. Therefore, we have the "coefficient of variation" of the stochastic variable $\frac{1}{N+1} \cdot\left\{\xi\left(B_{N+1}\left(b_{0}, F *(0, N ; \bar{D} ; \bar{D}, \xi)\right)\right)-\xi\left(b_{0}\right)\right\}$,

$$
\begin{aligned}
& \overline{U\left(F *(0, N ; \bar{D} ; \bar{D}, \xi) ; b_{0}, \xi\right)}= \frac{\sqrt{\left(R^{2}+\sigma^{2} \cdot \alpha^{2}\right)^{N+1}-R^{2 \cdot(N+1)}}}{R^{N+1}-1} \\
&= \frac{\sqrt{\left(1+\sigma^{2} \cdot \alpha^{2} / R^{2}\right)^{N+1}-1}}{1-1 / R^{N+1}} \\
&(R=1+r+(m-r) \cdot \alpha) .
\end{aligned}
$$

Hence, if $N$ becomes positive infinite, the "coefficient of variation," (9.8), also becomes positive infinite in S.D.M.S.-I, since $\sigma>0, \alpha>0$ and $R=1+r+(m-r) \cdot \alpha$ $>1$ hold true. That is, if a linear utility function of amount of money is employed, the expectation of the utility of amount of money has not any reliability in S. D. M.S.I, in the case of $N$ is sufficiently large.

(B) Next, Bernoulli [4] proposed to employ a logarithmic function

$$
\xi(e)=A \cdot \log e+L
$$

as a utility function of amount of money, in order to evade a contradiction due to stochastic strategy-making which maximizes simple mathematical expectation of amount of money. And if Bernoulli's utility function is applied in the criterion functional (4.4) in the case of $n=N$, the criterion functional is denoted by 


$$
\begin{aligned}
& U\left(F\left(N, N ; b_{N}\right) ; b_{N}, \xi\right) \\
& \quad=A \cdot \underset{\psi_{N}}{E}\left[\log \left\{\left(b_{N}+K / r\right) \cdot\left(1+r+\left(C_{N}-r\right) \cdot h_{N}\right)-K / r\right\}-\log b_{N}\right] .
\end{aligned}
$$

Now, if $K>0$, there is a $b_{N}$-feasible strategic function $F\left(N, N ; b_{N}\right)$ with respect to any assigned $b_{N}$ such that $-K / r \leqq b_{N} \leqq 0$. However, the criterion functional $(9.10)$ cannot measure degrees of effectiveness of the $b_{N}$-feasible strategic function $F\left(N, N ; b_{N}\right)$ in the case of $-K / r \leqq b_{N} \leqq 0$, since $\log b_{N}$ cannot be defined.

Moreover, if $K<0$, the utility function $\xi(e)=A \cdot \log e+L$ may be approximated to a linear function even in the neighbourhood of $e=-K / r$. And if $b_{0}+K / r$ is a sufficiently small positive number, the domain $D\left(B_{N+1}\left(b_{0}, F\left(0, N ; b_{0}\right)\right)\right.$ may be also in the neighbourhood of $b_{0}=-K / r$, even in the case of $N$ is a large integer. In such a case, the Bernoulli's utility function will produce such a contradiction as we saw in a stochastic strategy-making which maximizes mathematical expectation of amount of money (i.e. employs a linear utility function of amount of money).

After all, Bernoulli's utility function can be applied only in the case of $K=0$, in S. D. M. S.-I.

\section{Bibliography}

[1] Arrow, K. J.: “Utilities, Attitudes, Choices," A Review Nort, Econometrica, Vol. 26, No. 1, January, 1958.

[2] Bellman, R.: Dynamic Programming, New Jersey ; Princeton University Press, 1957.

[3] - : "On the Theore of Dynamic Programming-A Warehowsing Problem," Management Science, April, 1956.

[4] Bernoulli, D.: "Exposition of a New Theory on the Management of Risk," Econometrica, Vol. 22, 1954.

[5] Charnes, A., Drezze, J. and Miller, M.: "Decision and Horizon Rules for Stocnastic Planning Problems: A Linear Example," Econometrica, Vol. 34, No. 2, April, 1966.

[6] Chipman, J.S.: “The Foundation of Utility," Econometrica, Vol. 28, April, 1960.

[7] Dantzig, G. B. and Madamsky, A.: “On the Solution of Two-stage Linear Programms under Uncertainty," Preceedings of the 4th Symposium on Mathematical Statistics and Probability, Vol. 1, University of California, Berkeley, 1961.

[8] Friedman, M. and Savage L. J.: "The Utility Analysis of Choice Involving Risk," Journal of Political Economy, 56, 1948.

[9] Hicks, L.R.: “Liquidity," Economic Journal, Dec., 1962.

[10] KugimiYa, Y.: “On the Stochastic Dynamic Programming for an Independent, Stationary, Stochastic Process," Bulletin af Mathematical Statistics, Vol. 11, No. 1 2, 1964.

[11] Luce, D. and RAifA, H.: Game and Decision, New York: John Wiley and Sons, Inc., 1957.

[12] Marschak, J.: "Rational Behavior Uncertain Prospects and Measurable Utility," Econometrica, Vol. 18, 1950.

[13] Mosteller, F. and Nogee, P.: “An Experimental Measurement of Utility," Journal of Political Economy, 59, 1951.

[14] Murphy, R.E., Jr.: Adaptive Processes in Economic Systems, New York and London, Academic Press, 1965.

[15] Neumann, J.V. and Morgenstern, O.: Theory of Game and Economic Behaviour, 2nd ed., Princeton University Press, 1947.

[16] Savage, L. J.: Foundation of Statistics, New York, John Wiley and Sons, Inc., 1954.

[17] Simon, H.A.: "Dynamic Programming under Uncertainty with a Quadratic Criterion Function," Econometrca, January, 1956. 